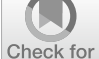

updates

Cite as

Nano-Micro Lett.

(2020) 12:126

Received: 2 April 2020

Accepted: 19 May 2020

Published online: 13 June 2020

(C) The Author(s) 2020

\section{Opportunities and Challenges in Twisted Bilayer Graphene: A Review}

\author{
Amol Nimbalkar ${ }^{1}$, Hyunmin $\operatorname{Kim}^{1} \bowtie$ \\ $\triangle$ Hyunmin Kim, hyunmin.kim@dgist.ac.kr \\ 1 Division of Biotechnology, Daegu Gyeongbuk Institute of Science and Technology (DGIST), Daegu 42988, \\ Republic of Korea
}

\title{
HIGHLIGHTS
}

- This article presents an overview of twisted bilayer graphene (tBLG) on their fabrication techniques and twisting angle-dependent properties.

- The properties of tBLG can be controlled by controlling the twisting angle between two graphene sheets.

ABSTRACT Two-dimensional (2D) materials exhibit enhanced physical, chemical, electronic, and optical properties when compared to those of bulk materials. Graphene demands significant attention due to its superior physical and electronic characteristics among different types of $2 \mathrm{D}$ materials. The bilayer graphene is fabricated by the stacking of the two monolayers of graphene. The twisted bilayer graphene (tBLG) superlattice is formed when these layers are twisted at a small angle. The presence of disorders and interlayer interactions in tBLG enhances several characteristics, including the optical and electrical properties. The studies on twisted bilayer graphene have been exciting and challenging thus far, especially after superconductivity was reported in $\mathrm{BLLG}$ at the magic angle. This article reviews the current progress in the fabrication techniques of twisted bilayer graphene and its twisting angle-dependent properties.

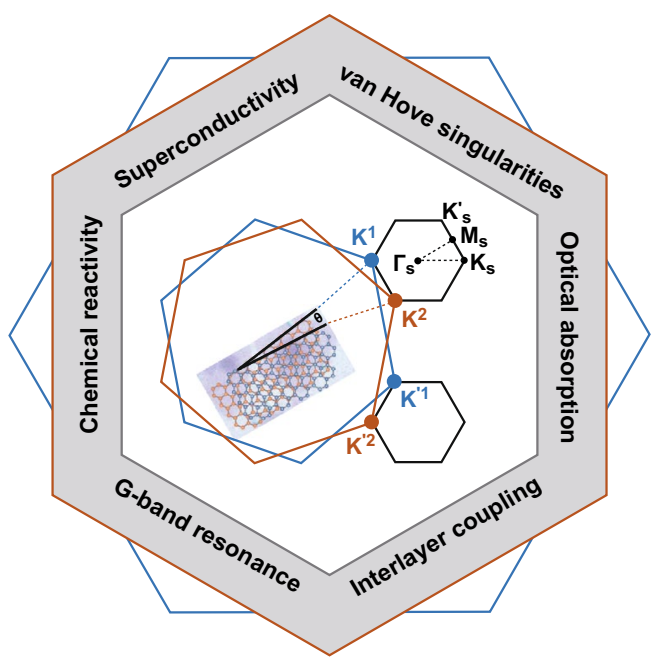

KEYWORDS Graphene; Twisted bilayer graphene; Magic angle; Superconductivity; van Hove singularities

\section{Introduction}

Graphene is composed of a one-atom-thick $s p^{2}$ hybridized allotrope of carbon atoms, which takes the form of a twodimensional (2D) planar honeycomb lattice. It has attracted abundant interest after its first isolation was achieved through the micromechanical cleavage of graphite in 2004 [1-4]. It has been seen as a promising material for applications in sensor, photonic, and electronic devices because of its excellent properties, such as chemical stability, high carrier mobility, low density, and optical transparency [5-9]. However, contrary to the single-layer graphene (SLG), while combining two or more layers of 2D materials in a specific order to fabricate the multilayer structures [10], their mechanical, optical, and electronic properties might be manipulated by varying the stacking order, interlayer spacing, and relative twisting angle [11-16]. The bilayer graphene (BLG) is a simple multilayer structure; in the simple form, two graphene layers ordered 
in an $\mathrm{AB}, \mathrm{AA}$, or a twisted orientation [17-19]. The practical assemblage of distinct graphene layers to make bilayer graphene infrequently leads to an impeccable stacking order; thus, small twisting of a single graphene layer is observed relative to the other [20], which modifies the electronic properties [10, 20,21]. The atomic orientation among the two layers might further vary, as the bilayer graphene has a weaker interlayer van der Waals bonding due to the lattice deformation, which intensely affects the interlayer electron motion [22-27].

Twisted bilayer graphene (tBLG) is fabricated by the stacking of two monolayers of graphene with a specific twisting angle $(\theta)$ [28]; in this structure, the moiré pattern has been observed to emerge with a higher periodicity [19, 29]. Recently, the tBLG form of graphene attracted significant attention from several researchers both theoretically as well as experimentally due to its extraordinary optical [30-33] and electronic properties [33-38], which were a result of the development of the moiré patterns. The tBLG systems show the Dirac spectra with twisting angle-dependent (analogous to chirality dependence in carbon nanotube systems) lower-energy van Hove singularities (vHSs), Fermi velocity, magnetoresistance oscillations, and quantum Hall effect (QHE) [6, 17, 18, 21, 39-41]. Therefore, the progress of tBLG-based devices shows excessive potential due to the tunable interlayer coupling and band structure of tBLG $[17,28]$. This interest arises predominantly from the physics of tBLG due to the presence of low-lying flat bands near the magic angles [42]. The Fermi energy of the tBLG structure is less than $10 \mathrm{meV}$ near the magic angle $\left(\theta=1.05^{\circ}\right)$ [43]; however, upon comparison, the superconductor critical temperature $\left(T_{\mathrm{c}}\right) \sim 1 \mathrm{~K}$ is comparatively high [44]. Meanwhile, several theoretical studies reported the understanding of the superconductor and insulator phases of the tBLG [44-65]. In this review article, we aim to highlight the current progress in the fabrication of tBLG systems as well as their properties and expect to motivate more researchers to work on tBLG systems for different applications.

\section{Synthesis Methods}

In this section, we reviewed some of the recent advanced synthesis methods used to fabricate twisted graphene sheets. The twisted graphene layers are formed on the surface of crystalline graphite naturally due to the accidental folding of graphene layers onto other graphene flakes or themselves [66, 67]. Several techniques have been reported for preparing graphene sheets, such as chemical exfoliation from bulk graphite [68], micromechanical cleavage of highly ordered pyrolytic graphite (HOPG) [69], chemical reduction of chemically exfoliated graphene oxide [70], solid-state graphitization or thermal decomposition of $\mathrm{SiC}$ [71, 72], and thermal and plasma-enhanced chemical vapor deposition [73, 74]. Every technique has its own set of advantages and disadvantages. The usually followed method is based on the stacking of two SLGs synthesized by chemical vapor deposition (CVD) to fabricate twisted graphene [75], as shown in Fig. 1a. It usually includes the transfer of CVD-grown single-layer graphene (SLG) onto $\mathrm{SiO}_{2} / \mathrm{Si}$ substrates via a simple wet-chemistry method [76-78], with a protective layer of poly(methyl methacrylate) (PMMA) [78] followed by $\mathrm{Cu}$ etching. After the transferring of SLG/PMMA onto the substrate, the PMMA is removed by using acetone and annealing [77]. The twisted bilayer graphene samples are prepared by consecutively transferring a second SLG onto the $\mathrm{SLG} / \mathrm{SiO}_{2} /$ $\mathrm{Si}$ in the same way. The prepared sample is baked in the air at $150{ }^{\circ} \mathrm{C}$ for $20 \mathrm{~min}$ for enhancing the adhesion between the SLG substrate and SLG-SLG [75]. In this technique, the removal of PMMA is a crucial step because if residual PMMA is found at the top and bottom of SLG, it can affect the interlayer interaction among the graphene layers. The control of the twisting angle is difficult in this technique due to the random shapes of SLG grown by CVD.

In another report, Kim et al. [79] reported a new approach by demonstrating the preparation of a smalltwist-angle bilayer graphene by utilizing hexagonal boron nitride (hBN) and sequential graphene flake pickup steps by utilizing a hemispherical handle substrate [80], as shown in Fig. 1b. The rotationally aligned transfer process provides a superior control on crystal axes alignment, as well as on small twisting angle due to the controlled flake pickup and selective detachment from the exfoliation substrate. A single graphene flake is divided into two separate regions, as illustrated in Fig. 1b. The divided flakes are then successively picked up by an hBN flake attached to the hemispherical handle. Among the first and the second graphene flake pickup, the substrate is rotated by $0.6^{\circ}$ to $1.2^{\circ}$ twisting angle with a $0.1^{\circ}$ accuracy. The substrate is twisted by a diminutive angle among the two stages. The stem of two graphene flakes from the same graphene layer; a small twist angle is produced among the crystal axes of the distinct layers. The two SLGs are likely to have 
(a) Transfer \#1,
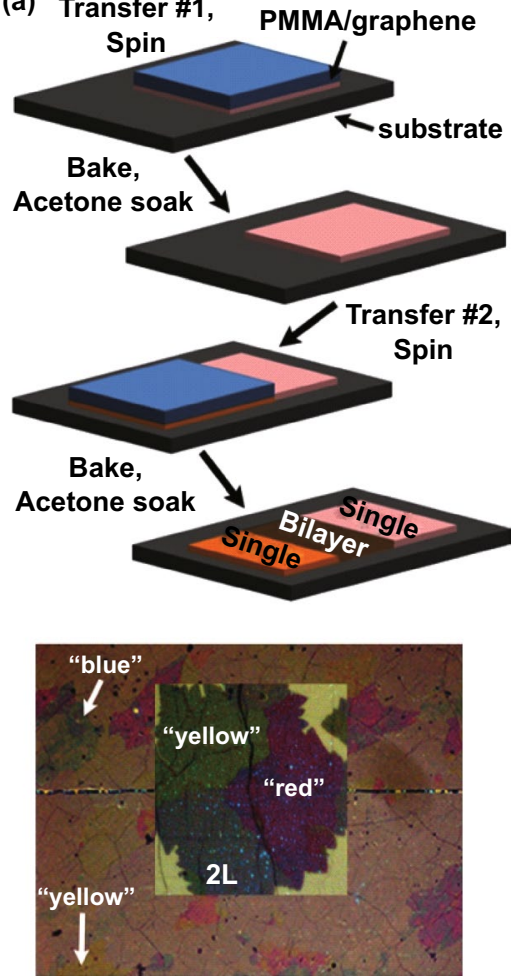
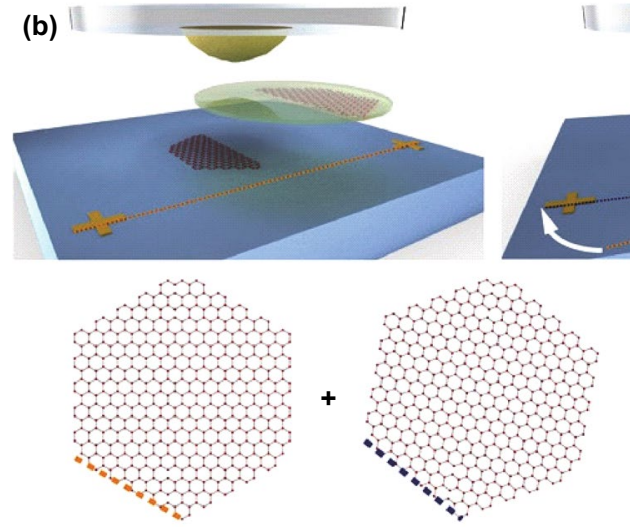

(c) $\theta_{1=13^{\circ}}$

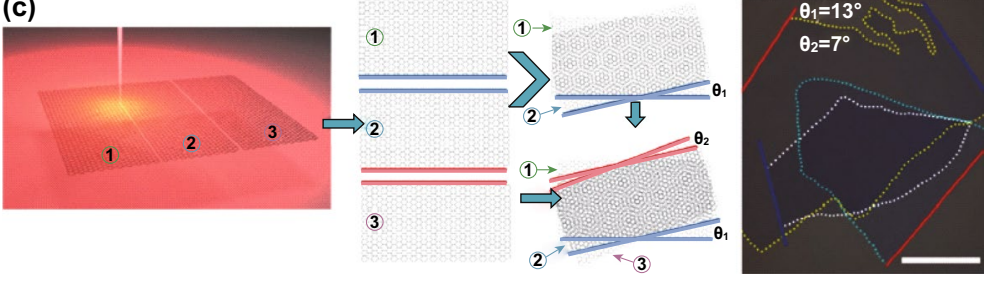

(d)

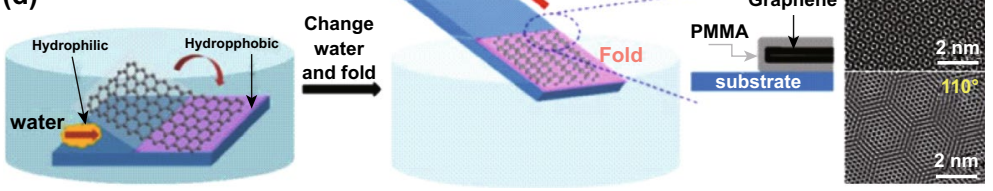

Fig. 1 a Schematic of the synthesis process flow of twisted bilayer graphene (tBLG) by CVD and optical microscope image of tBLG on $\mathrm{SiO}_{2} /$ Si substrate [75]. b Schematic illustration of detaching an SLG using a hemispherical handle and schematic illustration of the moiré pattern formation [79]. $\mathbf{c}$ The schematic of the CRS method utilized to fabricate tBLG, double twisted trilayer graphene (DTTG), and optical microscopy image of DTTG [81]. d The schematic of the folding procedure of a single-layer graphene sheet with folding driven by delamination only from

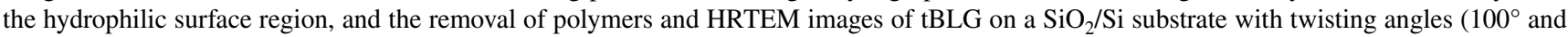
$110^{\circ}$ ) [82]. Adapted with permission from Refs. [75, 79, 81, 82]

rotationally aligned crystal axes ensuring an exact tBLG structure because of the graphene single-crystal nature. This fabrication method fulfills the requirements of scientific research and applications due to precise control over the twisting angle. In another report, Chen et al. [81] prepared tBLG by the cutting, rotating, and stacking (CRS) of the graphene layer using femtosecond laser micromachining and a precise transfer method. The single-layer graphene was mechanically exfoliated on a $\mathrm{Si} / \mathrm{SiO}_{2}$ substrate using a femtosecond laser and was divided into two pieces with a pair of parallel and straight cutting lines, as represented in Fig. 1c. By using the two cutting lines, the two graphene sections were rotated with an angle $\theta$ and were accurately stacked together onto to $\mathrm{Si} / \mathrm{SiO}_{2}$ substrate using a suitable transfer method. The control over the twisting angle of tBLG fabricated by this method is better than the other preparation methods.
Wang et al. [82] prepared tBLG films based on the controlled folding of single-layer graphene. There are three steps in this method: (1) the transformation of the $\mathrm{SiO}_{2} /$ Si substrate to make hydrophilic and hydrophobic sections with a distinct border, (2) the controlled SLG/PMMA delamination from the hydrophilic section in water, and (3) removal of the PMMA layer. The controlled folding in this procedure allows for the fabrication of tBLG structure with distinct stacking orientations; the required twisting angle was achieved by varying the hydrophilic and hydrophobic boundary folding angles. In a recent report, Cao et al. [43] fabricated tBLG by the vertical stacking of graphene to study its superconductivity properties at $1.05^{\circ}$ and $1.16^{\circ}$ magic angles. In this method, one piece of SLG is fixed, and the other is stacked vertically by the mechanical transfer method on the fixed one at different angles. The vertical 
stacking method makes it simple and easy to achieve the desired twisting angle in tBLG.

\section{Lattice and Electronic Structure of tBLG}

The variation in the graphene properties is produced due to the mode of stacking orientation among the two graphene layers and the number of stacked graphene layers [83, 84]. The bilayer graphene is fabricated by the vertical stacking of two graphene layers, which results in the breaking of the symmetry of bilayers. The broken symmetry causes inequivalent charge and electrostatic potential among the two graphene layers [85]. However, the tBLG is non-ABstacked bilayer graphene, in which one graphene sheet rotates by a definite angle $(\theta)$, as compared to the other [86]. The schematics of band structure and the equivalent density of state (DOS) with van Hove singularities (vHSs) in tBLG are shown in Fig. 2a [86]. The Dirac band dispersions vary drastically and become strongly warped with smaller twisting angles (less than $5^{\circ}$ ) [87-89]. The Dirac cones of the two distinct layers intersect and produce saddle points in the shared space of tBLG [90], causing the development of van Hove singularities (vHSs) in the density of state (DOS) [91-93], which enhances the Raman $G$ band resonance and optical absorption and improves the chemical reactivity of tBLG [93-97]. The band structure of the tBLG domain where the band dispersions are cutting across (Fig. 2b), and the constant-energy contours to the two neighboring Dirac points (Fig. 2d) [86], confirms the formation of the vHSs by the intersection of two Dirac cones. The existence of logarithmic vHSs was also confirmed by Brihuega et al. [98] by using local DOS (LDOS) of two stacked graphene layers with twisting angles between $1^{\circ}$ and $10^{\circ}$, as shown in Fig. 2c. The small twist angles in bilayer graphene executed a pattern on the intercalated atoms that causes the modification of the electronic properties (Fig. 2e), which is used as a high-mobility material with significant bandgaps [99]. The moiré patterns are produced through the graphene-graphene interaction by matching the relative orientations of the top-layer graphene lattice, as shown in Fig. 2f. The moiré lattice and strong non-perturbative characteristics of the $\mathrm{tBLG}$ in the smaller twisting angle regime are confirmed by Wong et al. [100]. The simplest tBLG also shows unique physical properties because of the controlled interaction among the two Dirac electron gases with a broad moiré pattern [101]. The tBLG with a smaller twisting angle is attended with a moiré pattern that produces particular super-periodicity and uniformity for the electron systems, which causes a substantial variation in the electronic properties $[102,103]$.
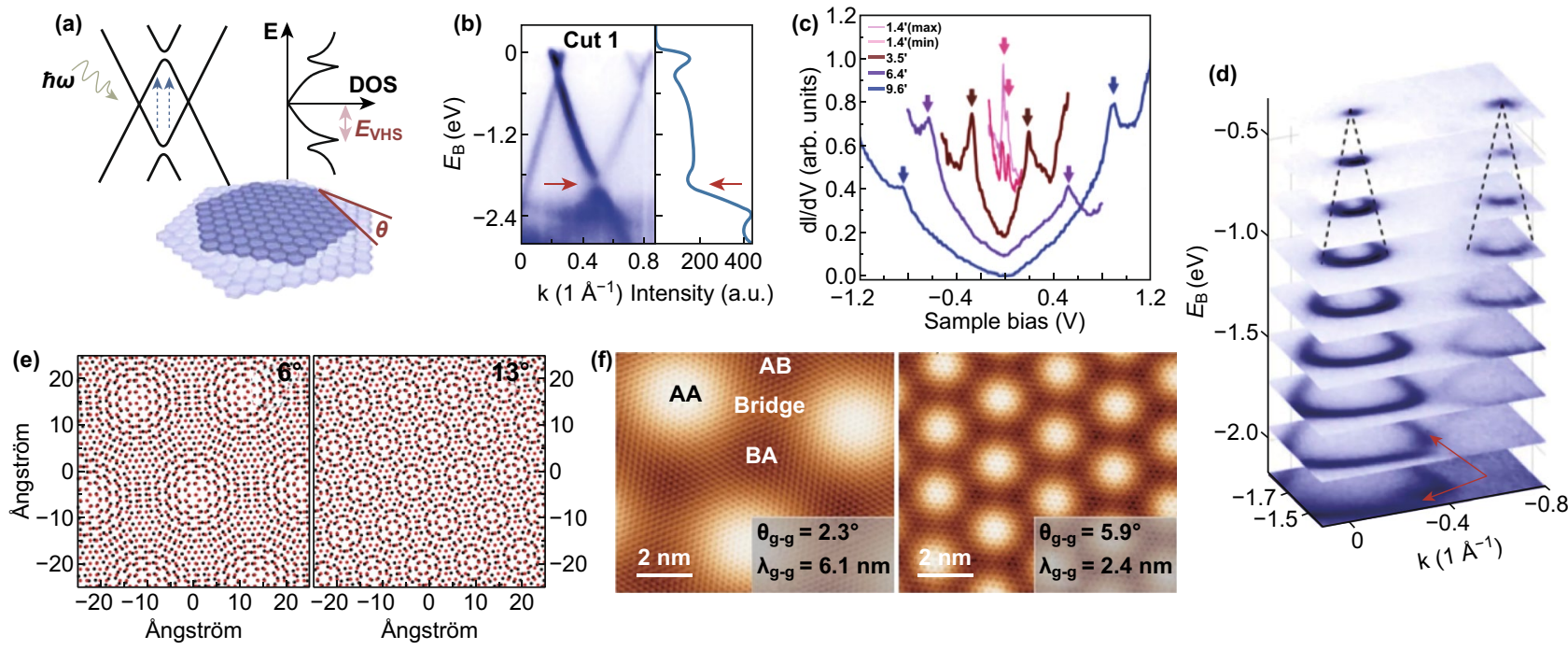

Fig. 2 a Schematics for band structure with mini-gaps and the corresponding density of state (DOS) with van Hove singularities (vHSs) in tBLG, b ARPES spectrum of tBLG [86]. c LDOS spectrum of moiré patterns with different twisting angles [98]. d Stacking plot of constantenergy contours at different binding energies of tBLG [86]. e Moiré patterns of tBLG with $6^{\circ}$ and $13^{\circ}$ twisting angle [99], f STM images of tBLG with $2.3^{\circ}$ and $5.9^{\circ}$ twisting angle [100]. Adapted with permission from Refs. [86, 98-100] 


\section{Raman Spectroscopy}

Raman spectroscopy plays a crucial role in the nondestructive analysis of the lattice structure, as well as the optical, electronic, and phonon properties of graphene [104, 105]. Raman spectroscopy is widely utilized to investigate the physical properties of graphene and graphene-based devices [101]. The Raman features dependent on the stacking orientation provide detailed information about the phonon characteristics with their distinctive band structures [106, 107]. The phonon vibration modes of the AB-stacked MLG are separated into an out-plane shear $(C)$ vibration modes and in-plane $G$ and 2D vibration modes. The first-order Raman endorsed peak in graphene is a $G$ band observed at $\sim 1584 \mathrm{~cm}^{-1}$, accomplishing momentum conservation, which commands that the scattered phonon must carry no momentum. The $D$ and $D^{\prime}$ bands are originated from the intervalley and intravalley double-resonance Raman scattering mechanisms, respectively [108-110]. Two additional $R$ and $R^{\prime}$ modes, which are from the TO and LO phonon branches, respectively [111-113], can differentiate the stacking orientation among the $\mathrm{AB}$ stacking and twist. The $\mathrm{tBLG}$ superlattice offers a $\theta$-dependent $q$ wave vector, which triggers phonons inside of the Brillouin zone, due to which the layer breathing vibrations ( $\mathrm{ZO}^{\prime}$ phonons) can be studied in a first-order light scattering [112]. Lui et al. reported that the Raman band is susceptible to interlayer interactions; it can reveal a distinctive line shape for the graphene band of every layer thickness and stacking order [114].

For investigating the twisting angle effects in tBLG, He et al. [115] carried out the Raman spectroscopy less than $100 \mathrm{~cm}^{-1}$ Raman shifts and observed two modes in a smaller range of twist angle. Figure 3 shows the $G$ Raman peak intensity is intensely enhanced as a function of twisting angle, which illustrates that these $G$ Raman modes and the lower-energy modes share the equivalent resonance amplification mechanism. The intensity of the enhanced $G$ peak and the 2D band displays a maximum blueshift than the SLG at $12^{\circ}$ twisting angle (called a critical angle) (Fig. 3a). The phonons above $100 \mathrm{~cm}^{-1}$ displayed by the Raman scattering process are intermediated by the superlattice wave vector $q$, which simply depends on the twist angle $(\theta)[116,117]$. The background-subtracted lower-energy Raman spectrum for different tBLG domains is shown in Fig. 3b. The observed fundamental layer breathing $\left(\mathrm{ZO}^{\prime}\right)$ and out-of-plane acoustic
(ZA) modes between 130 and $180 \mathrm{~cm}^{-1}$ are activated by the formation of moiré pattern $[110,117]$. The additional fundamental layer breathing mode $\left(\mathrm{ZO}^{\prime}\right)_{L}$ is observed at $\sim 94 \mathrm{~cm}^{-1}$. The intensities of $\left(\mathrm{ZO}^{\prime}\right)_{L}$ frequency mode, and the background covering on which the lower-energy Raman lines are comprised, demonstrate substantial resonance enrichment near the $12^{\circ}$ twisting angle; it is associated with the $G$ Raman peak enhancement. Another Raman mode observed at $\sim 52 \mathrm{~cm}^{-1}$ is ascribed to the twisting mode due to the rotation between the two graphene layers with respect to each other. The advancement of the frequency and FWHM of the $\mathrm{ZO}^{\prime}$ mode as a function of $L$ normalized $I_{2 \mathrm{D}}$ are shown in Fig. 3c, d. The $\left(\mathrm{ZO}^{\prime}\right)_{L}$ mode frequency increases with the $I_{2 \mathrm{D}}$ when the twisting angle is less than the critical angle, and the frequency becomes nearly constant, when the twisting angle is greater than the critical angle. This illustrates that the intense variations in the FWHM and frequency of the $\left(\mathrm{ZO}^{\prime}\right)_{L}$ mode occurred when the twisting angle is close to the critical angle $\left(12^{\circ}\right)$. The low-frequency phonon dispersion shown in Fig. 3e, which shows the phonon frequency observed at $\sim 94 \mathrm{~cm}^{-1}$, and the phonon wave vector $k_{\left(\mathrm{ZO}^{\prime}\right) L}$ are in good arrangement with the $\mathrm{ZO}^{\prime}$ phonon dispersion in tBLG and confirming the obligation of this mode with the layer breathing mode $\left(\mathrm{ZO}^{\prime}\right)_{L}$. The Raman study reveals that the fundamental properties of $\mathrm{tBLG}$ are different from the Bernal-stacked bilayer graphene.

The Raman imaging reveals the differences in interlayer interactions, which is useful for the investigation of external physical, chemical, and optical properties of bilayer graphene. The white light dissimilarity enhances the reflection image of single and bilayer graphene on $\mathrm{SiO}_{2}$ substrate which is shown in Fig. 4a. The bilayer graphene comes out darker than the single-layer graphene with homogeneous intensity. The dark-field TEM image of tBLG domains with different twisting angles is shown in Fig. 4b. The dissimilarities in Raman intensity are associated with the twisting angle, and within the same domain, both bands intensities are nearly constant. The Raman spectra of the same area show the $G$ band intensity increases for the $\sim 12^{\circ}$ twisting angle, attributable to distinctiveness in the joint density of states (JDOS) of tBLG, and its energy is entirely depending on the twisting angle, and the strength of optical transition is directed by the interlayer interactions, which allow direct optical imaging of these parameters, as shown in Fig. 4b. The 2D peak position, intensity, and width vary quickly around the $\sim 12^{\circ}$ twisting angle, which describes a transition 

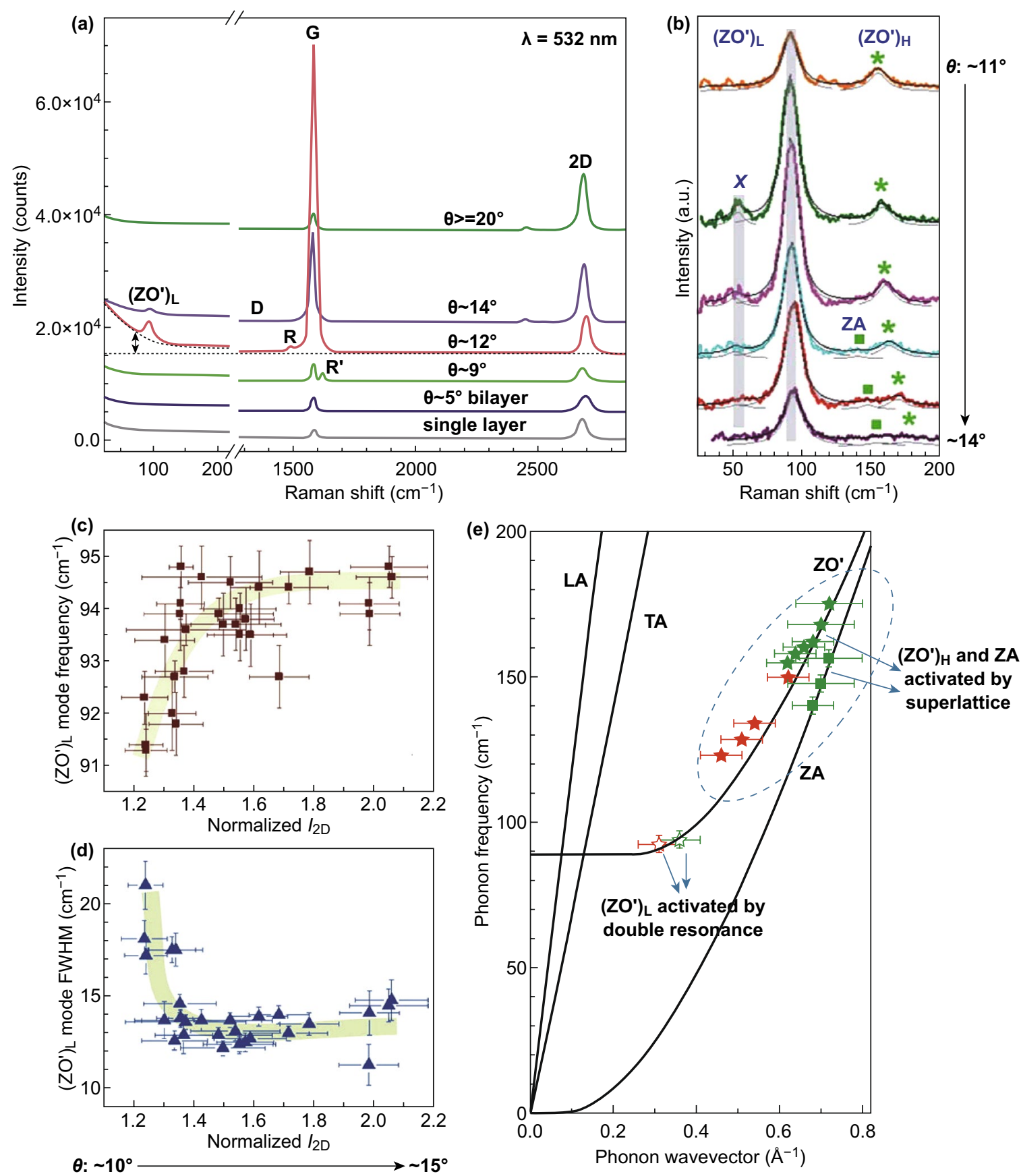

Fig. 3 a Raman spectrum of tBLG with different twisting angles excited by a $532 \mathrm{~nm}$ laser excitation. b Background-subtracted low-energy Raman spectrum of six tBLG domains, the gray vertical bars highlight $X$ and $\left(\mathrm{ZO}^{\prime}\right)_{L}$ modes, and the squares and the asterisks highlight $\mathrm{ZA}$ and $\left(\mathrm{ZO}^{\prime}\right) \mathrm{H}$ modes, respectively. $\mathbf{c}$ Frequency and $\mathbf{d} \mathrm{FWHM}$ of the $\left(\mathrm{ZO}^{\prime}\right)_{L}$ mode as a function of normalized $\mathrm{I}_{2 \mathrm{D}}$. e Low-frequency phonon dispersion [115]. Adapted with permission from Ref. [115]

among the low and high twisting angle regions. The widefield $G$ band images of the similar tBLG domain at different excitation energies and the plot of $E_{\mathrm{ex}}$ versus $\theta_{\text {peak }}$ are shown in Fig. 4d, which reveals the different domains and shows intensity enhancement for different twisting angles corresponding to different excitation energies. The twisting angle with specified excitation energy allows upcoming studies of electrical, mechanical, and optical properties of tBLG at precise angles. In the optical properties of $\mathrm{tBLG}$, parallel band optical transitions play a vital role, which might 

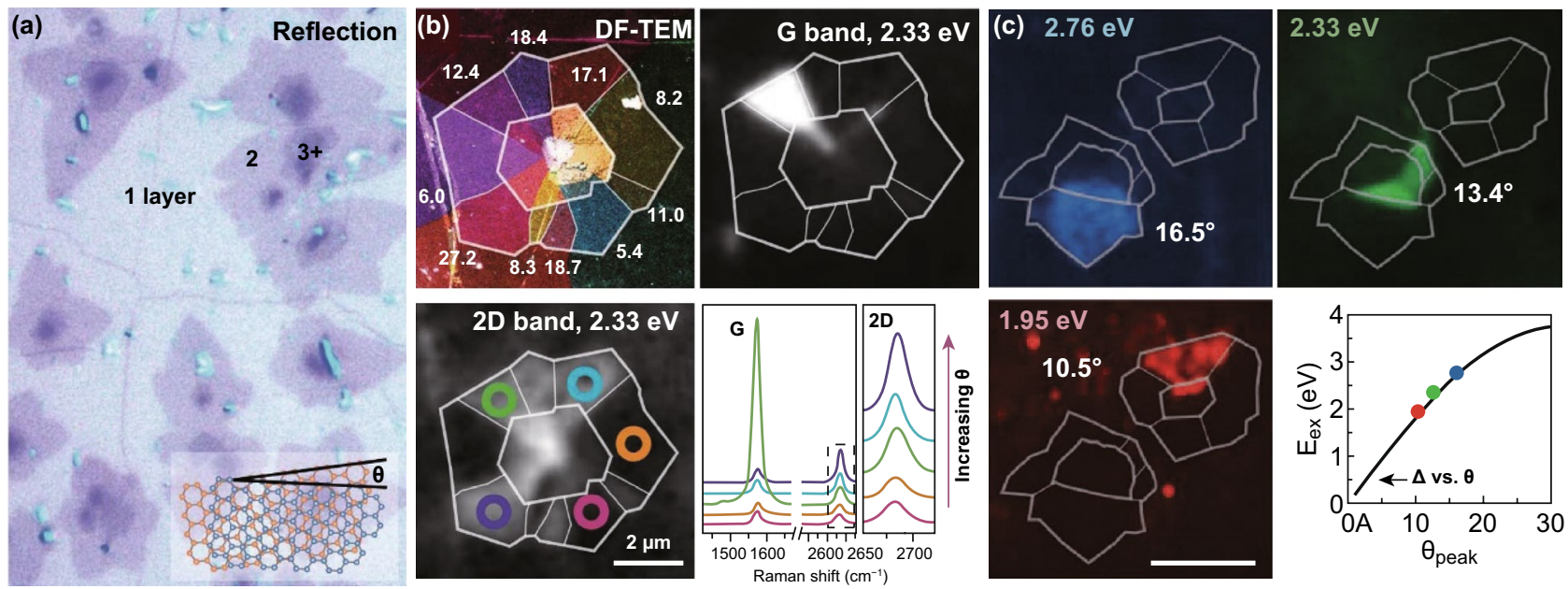

Fig. 4 a Optical reflection image of CVD-grown graphene transferred on $\mathrm{SiO}_{2} / \mathrm{Si}$, and inset shows structure of tBLG with a twisting angle $\theta$. b Dark-field TEM, $G$ band, and 2D band Raman images of the same tBLG domain. $\mathbf{c}$ Wide-field $G$ band images of the same tBLG section at three different excitation wavelengths with different $G$ band enhancement domains, plot of $E_{\mathrm{ex}}$ versus $\theta_{\text {peak }}$ [118]. Adapted with permission from Ref. [118]

be further employed in emerging innovative optoelectronic devices with exceptionally tunable features via controlling over the twisting angle [118].

\section{Optical Properties}

The decreasing in the twisting angle interference among the two lattice periods creates a moire pattern with a higher wavelength, where the properties of tBLG such as van Hove singularity and bandgaps seem to be present in the far-infrared region and the band velocity of the Dirac cone is considerably decreased [37, 119-122]. The optical absorption study is usually employed for graphene-based systems to examine the electronic structures [122-125]. Recently, Anh Le and Nam Do reported the optical properties of tBLG using a time evolution of states in real space [126]. The optical conductivity of some incommensurate and commensurate tBLG structures is shown in Fig. 5a, which clearly showed the conductivity structure in the infrared-red region. The curves of all groups with different twisting angles reveal that the optical conductivity of tBLG continuously varies with the twisting angle, but the distinctive structure of the twisted systems $\left(\theta=0.01^{\circ}\right.$ to $\left.0.2^{\circ}\right)$ is found in the lowerenergy range. The transition developments for the tBLG structures with $\theta=10^{\circ}$ are shown in the inset of Fig. 5a. The $\mathrm{W}$ shape DOS is slowly converted into the $\mathrm{U}$ shape by decreasing the twisting angle to zero, which illustrates that the commensurability among the two graphene layers does not play a crucial role in varying the optical and electronic properties. Yu et al. [127] reported the optical absorption spectrum of electrically gated tBLG. The optical conductivity $\sigma_{1}(\omega)$ spectra of tBLG with different twisting angles show two separate interband transitions (Fig. 5b): (1) The frequency-independent conductivity $2 \sigma_{\text {mono }}$ comes from the LB transition, and (2) the major absorption peak $\alpha$ originates from the transitions $\mathrm{vHs}_{2} \rightarrow \mathrm{BE}_{1}$ and $\mathrm{BE}_{2} \rightarrow \mathrm{vHs}_{1}[128$, 129]. Inset of Fig. 5b shows the blueshift of $\alpha$-peak toward the higher energy as the twisting angle increases [129]. The splitting of the absorption edge of $\sigma_{\mathrm{LB}}$ into two edges with various energies reveals that the Dirac cones of the bottom and top layers are transferred by varying the total energy [127]. The intensity and energy of peak $\alpha$ show variation with the gate bias, as the interlayer potential irregularity breakdowns the configuration of the band edge and vHs. The $V_{\mathrm{G}}$-driven variation of $\sigma_{1}(\omega)$ for the hole doping region is shown in Fig. 5c. The absorption edge of $\sigma_{\mathrm{LB}}$ in Fig. 5c demonstrates a significant expansion, as well as the shifting toward higher energy; it demonstrates the shifting of the $\alpha$-peak toward lower energy, whereas its intensity is decreased noticeably, which confirms the changing of the band structure of tBLG with the gating. The optical absorption spectra showed notable variations like the shifting of 

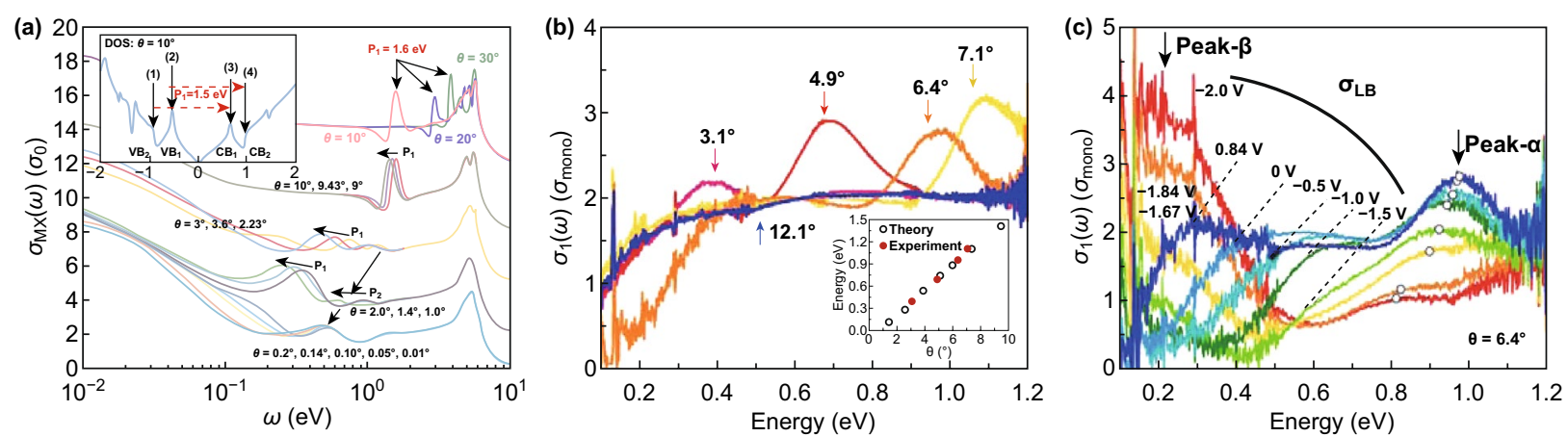

Fig. 5 a Optical conductivity of some commensurate and incommensurate tBLG structures, and inset shows the leading transition procedures contributing to the formation of the optical conductivity peaks [126]. b Optical conductivity $\sigma_{1}(\omega)$ of the different twisting angle tBLG samples, and inset shows comparison of the observed peak position and theoretical prediction. c Optical conductivity of gated tBLG $\left(\theta=6.4^{\circ}\right)$ for different gate voltages $V_{\mathrm{G}}$ [127]. Adapted with permission from Refs. [126, 127]

inter-van Hove singularity transition peak and the splitting of interlinear band absorption, as well as the appearance of an extremely strong intra-valence band transition.

The PL excitation spectrum recorded after 2-photon excitation, also the twisting angle-associated tunable linear absorption spectrum, is shown in Fig. 6a [130]. The resonant PL emission was positioned at around 2.0, 2.1 and $2.7 \mathrm{eV}$, as confirmed by applying $10 \mathrm{~nm}$ wide band-pass filters. The domain twisting angles were selected by 1-photon linear absorption spectrum, which spectrally overlaps with the consequent 2-photon PL excitation peaks. The splitting of 2-photon PL excitation and 1-photon absorption peak energies varies with the twisting angle. The PL map of tBLG structure collected at around $1.26 \mathrm{eV}$ excitation shows a significant PL emission enhancement after the 2-photon excitation of $17.5^{\circ}$ domains, as compared to the nearby domains (Fig. 6b) [130]. The appreciative band-pass optical filtering confirms the emission energy matches well with the 1-photon absorption resonance at $\sim 2.8 \mathrm{eV}$ of the $17.5^{\circ}$ domain. This resonant PL variation with the vHs reveals that the electrons will thermalize quickly to low metallic continuum states by electron-electron scattering. The line scanning results of the different graphene films by two different lasers wavelength (633 and $514 \mathrm{~nm}$ ) are shown in Fig. 6c [81]. The signal is increased by $35 \%$ for a $13^{\circ}$ twisting angle tBLG domain with $514 \mathrm{~nm}$ laser wavelength, and a $10^{\circ}$ twisting angle tBLG domain shows similar results for a $633 \mathrm{~nm}$ laser wavelength. The photocurrent of tBLG films might be extensively enhanced by both $s$ - and $p$-polarized lights with consequent laser wavelength consistent with the absorption enhancement, as shown in Fig. 6d [81]. The photocurrent and optical absorption enhancement of tBLG could be achieved by varying the twisting angles.

Recently, Yin et al. [86] reported a strong light-matter interaction and selectively improved photocurrent generation of tBLG devices under laser illumination for different twisting angles. The schematics of two parallel tBLG films with $13^{\circ}$ and $7^{\circ}$ twisting angle on $\mathrm{a} \mathrm{SiO}_{2} / \mathrm{Si}$ substrate embedded with two terminals are shown in Fig. 7a, b. The intensity of $G$ band of $13^{\circ}$ twisted tBLG domain showed a consistent 20-fold enrichment compared with the $7^{\circ}$ twisted tBLG domain, as shown in Fig. 7c. The interfacial junctions of tBLG metal electrodes were utilized to separate the photo-excited electrons and holes on the tBLG domain under the laser light $[131,132]$. The $13^{\circ}$ and $7^{\circ}$ twisted tBLG domains produce distinct photocurrent shifts, as shown in Fig. $7 \mathrm{~d}$. The $13^{\circ}$ and $7^{\circ}$ twisted tBLG domains produce 0.63 and $0.097 \mathrm{~mA}$ net photocurrent, respectively, at zero bias with a $532 \mathrm{~nm}$ laser. The net photocurrent mapping of the tBLG devices shows converse directions at two graphene electrode interfaces; for example, the intensity of photocurrent of $13^{\circ}$ twisted tBLG domain is $\sim 6.6$ times greater than the $7^{\circ}$ twisted tBLG domain (Fig. 7e, f). The photocurrent from both $13^{\circ}$ and $7^{\circ}$ twisted tBLG domains increases as the incident $532 \mathrm{~nm}$ laser power increases from $\sim 1 \mu \mathrm{W}$ to $\sim 5 \mathrm{~mW}$, as shown in Fig. $7 \mathrm{~g}$. The substantial enhancement in photoresponsivity of $13^{\circ}$ twisted tBLG domain below the enlightenment of different incident $532 \mathrm{~nm}$ laser power is observed. This twisting angle-dependent photocurrent enhancement holds enormous promise for high-selectivity photodetection applications. 


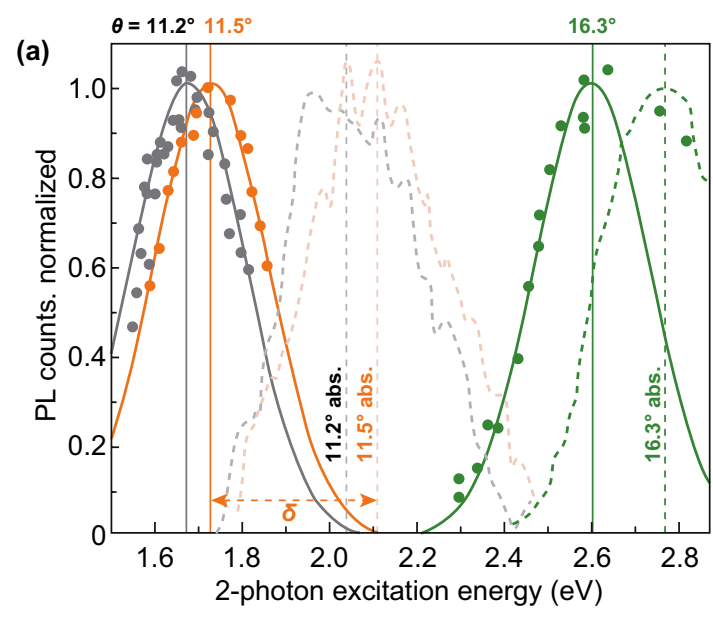

(b)

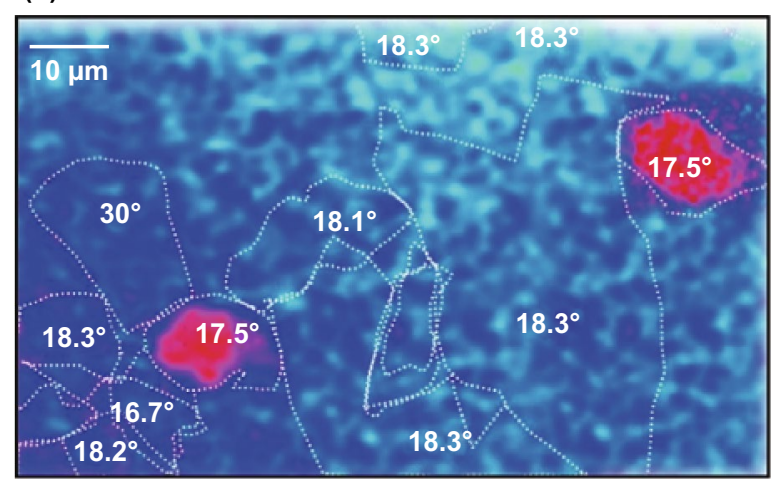

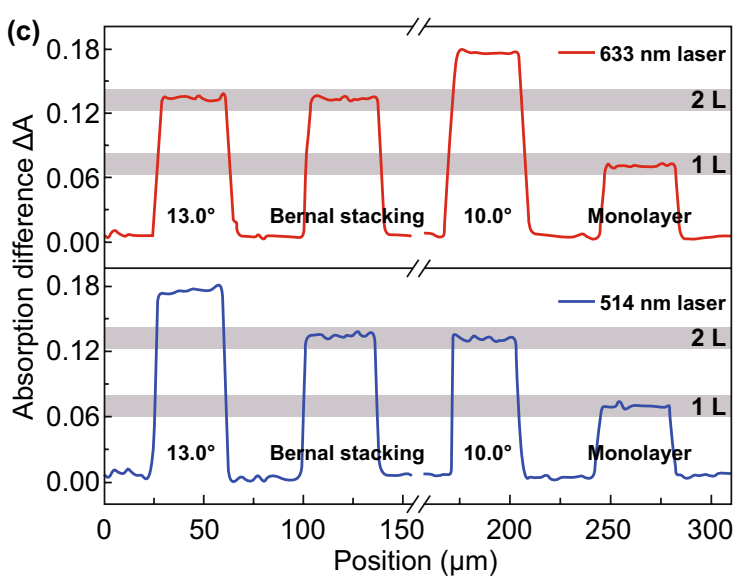

(d)

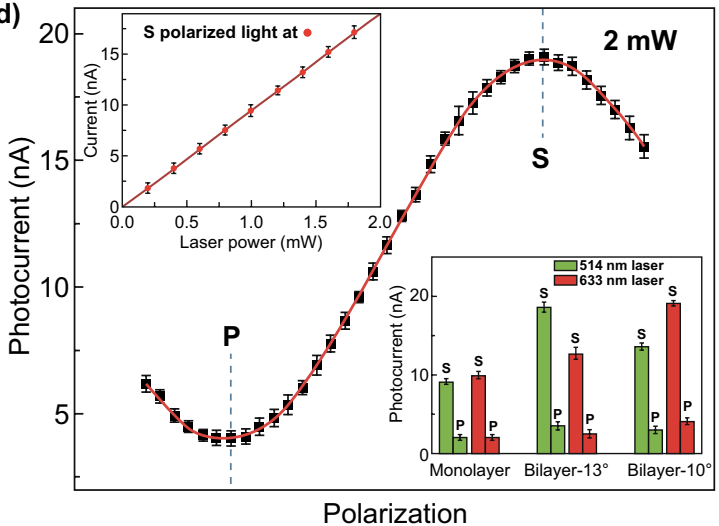

Fig. 6 a PL excitation peaks tune with $\theta=11.2^{\circ}, 11.5^{\circ}$, and $16.3^{\circ}$. b Scanning PL map of tBLG collected at $1.26 \mathrm{eV}$ excitation [130]. c Line scanning results of the graphene films for different laser energies. $\mathbf{d}$ The relationship between the incident light polarization and photocurrent and the upper inset image demonstrates the different laser powers and detected photocurrent, the photocurrent of the samples with different twist angles in the lower inset [81]. Adapted with permission from Refs. [81, 130]

\section{Electronic Properties}

The perfect and superior characteristics of the bilayer graphene in twisted multilayer graphene (tMLG) than the suspended form of graphene could be attributed to the fact that the tMLG is tens of nanometer in thickness and maintains the graphene layers ultra-clean, as well as free from any substrate influence. These extraordinary properties in tMLG generate from the higher degree of decoupling that occurs from the angular twisting between the layers [133]. Recently, Mogera et al. [134] reported the semiconducting to metallic transition converging behavior of twisted multilayer graphene (tMLG). The temperature-dependent conductivity $(\sigma)$ of the tMLG device in the $90 \mathrm{~K}$ to $273 \mathrm{~K}$ temperature range is shown in Fig. 8a. As the temperature increases, the conductivity per layer in the tMLG slowly increases and reaches a maximum at around $180 \mathrm{~K}$ and then linearly decreases up to $300 \mathrm{~K}$, which reveals the variation in non-monotonous conductivity with a distinctive semiconducting to metallic conversion on raising the temperature. The sequential difference in device photocurrent under light exposure is shown in Fig. 8b. The photocurrent increases in the semiconducting region and drops with the rising temperature in the metallic region and decreases with no photoresponse at a transition temperature (Fig. 8c). These pristine properties reveal the decoupled nature of the graphene layers in tMLG.

The interlayer contact conductance among the BLG with different twisting and stacking structures synthesized by the CVD method is recently discussed by Yu et al. [135]. A statistical method is applied for comparing the twisting angle-dependent current in the tBLG domains. The statistical results for different tBLG domain with various twisting 
(b)

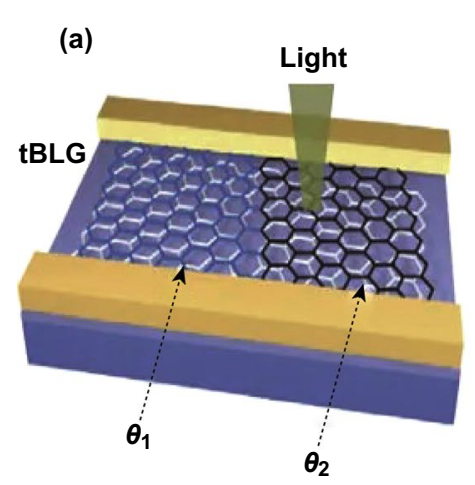

(c)
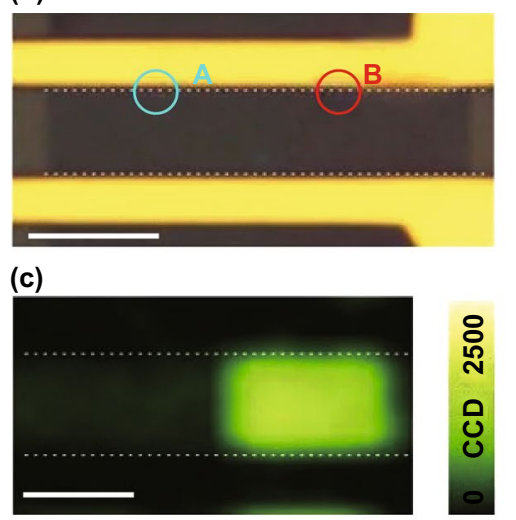

(d)

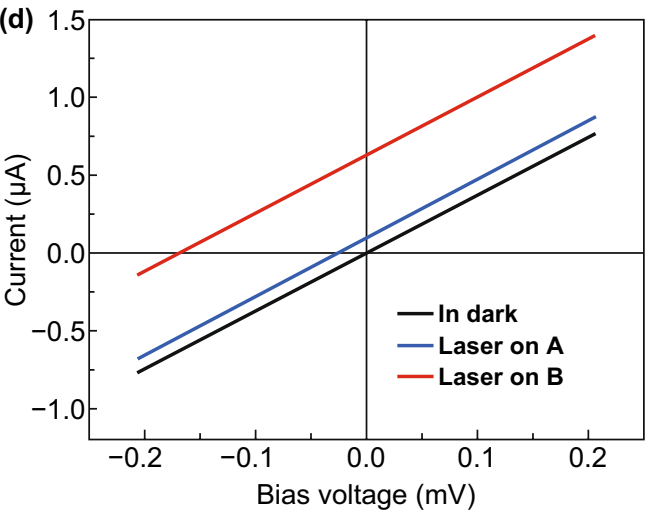

(e)

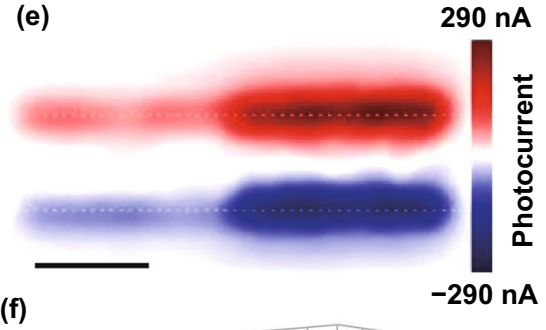

(f)

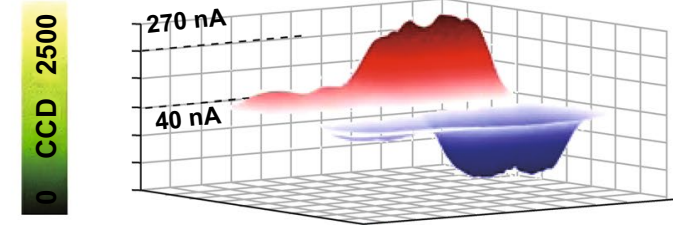

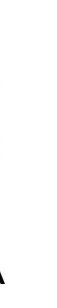 \\ .}

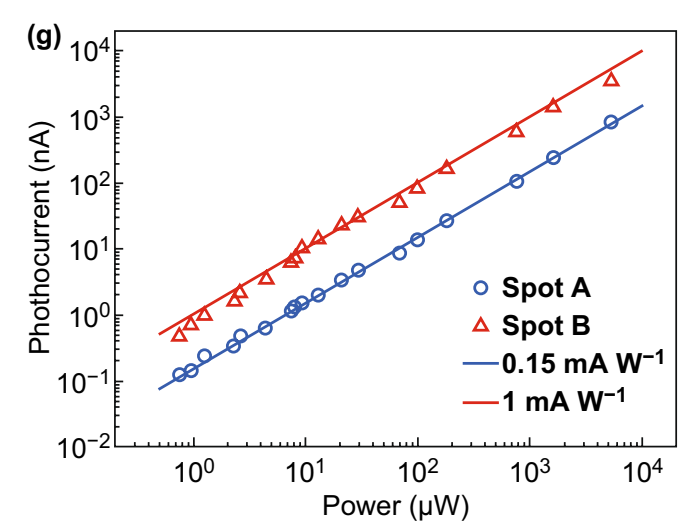

Fig. 7 a Schematic representation of a tBLG photodetection device, which comprises of two adjacent tBLG domains with different twisting angles of $7^{\circ}$ and $13^{\circ}$. b Optical image of the tBLG photodetection device. c Raman $G$ band intensity mapping image of $13^{\circ}$ twisting angled tBLG with an enhanced $G$ band intensity at $2.33 \mathrm{eV}$ laser energy. d Current versus source-drain bias curve with laser focus on spot A of $7^{\circ}$ and spot B of $13^{\circ}$ twisting angle in $\mathrm{tBLG}$ with laser off. e, f Scanning photocurrent image and its $3 \mathrm{D}$ view of the tBLG device. $\mathbf{g}$ Photocurrents generated as a function of incident power at spot A of $7^{\circ}$ and spot B of $13^{\circ}$ twisting angle of the tBLG device [86]. Adapted with permission from Ref. [86]

angles are shown in Fig. 8d. The tBLG with a small twisting angle displays a higher current, which indicates excellent contact conductance at no twisting among the graphene layers. Figure $8 \mathrm{e}$ shows the interlayer conductance of tBLG with various twisting angles. The interlayer contact conductance decreases with an increase in the twisting angle. The twisting angle propagation to the interlayer potential energy enhances, at the larger twisting angle [136]. The interlayer contact conductance of $0^{\circ}$ BLG domain is $\sim 4$ times higher than the $30^{\circ}$ tBLG domain, which reveals the twisting angledependent graphene interlayer contact conductance originated from the decoupling and coupling transitions.

Polshyn et al. [137] discussed the electrical transport measurements for different tBLG devices with $0.75^{\circ}$ to $2^{\circ}$ twisting angles in the room temperature. The resistivity $(\rho)$ of the tBLG domain $\left(\theta=1.06^{\circ}\right)$ measured near the flat band condition for carrier densities spanning the lower-energy band is shown in Fig. 8f, g. The resistance peaks or insulating phases at some integer multiples of $n_{\mathrm{s}} / 4$ and superconducting states at different partial band fillings are revealed in Fig. 8f. At nearly all densities, the resistivity $(\rho(T))$ increases with the increase in temperature and remains steady with the metallic behavior. The resistivity $(\rho(T))$ measured in tBLG devices with different twisting angles near $-n_{\mathrm{s}} / 2$ is shown in Fig. 8h. The resistivity is enhanced sub-linearly with the increase in temperature and reaches the highest point at a temperature $T_{\mathrm{H}}$; the resistivity scales linearly with temperature below the temperature $T_{\mathrm{H}}$. At the lowest temperatures, resistivity diverges from a linear dependence on temperature. The tBLG devices clearly show the resistivity saturation, superconducting, or insulating behavior at moderate temperature regimes. The observed three distinctive 

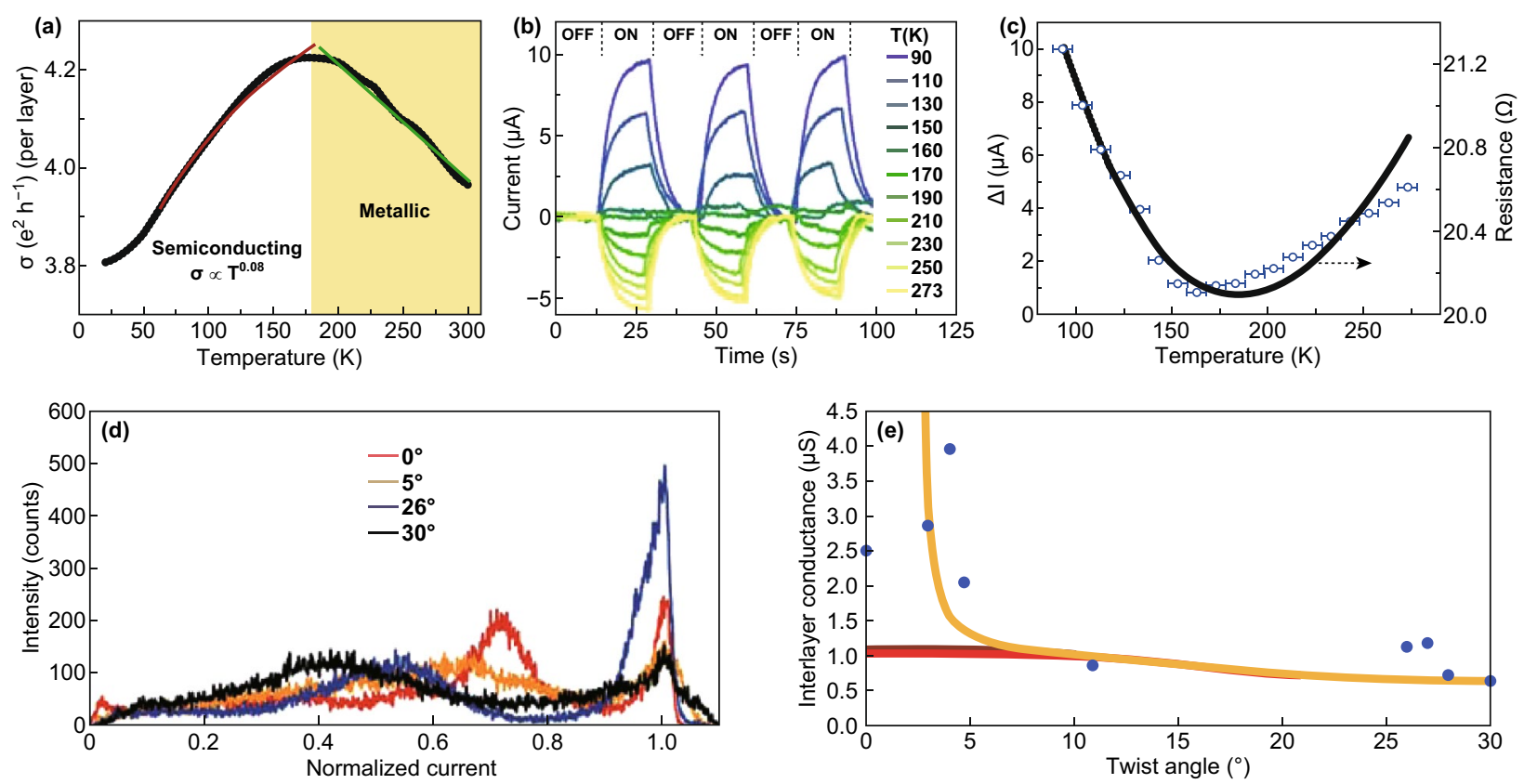

$0 \quad 1020$
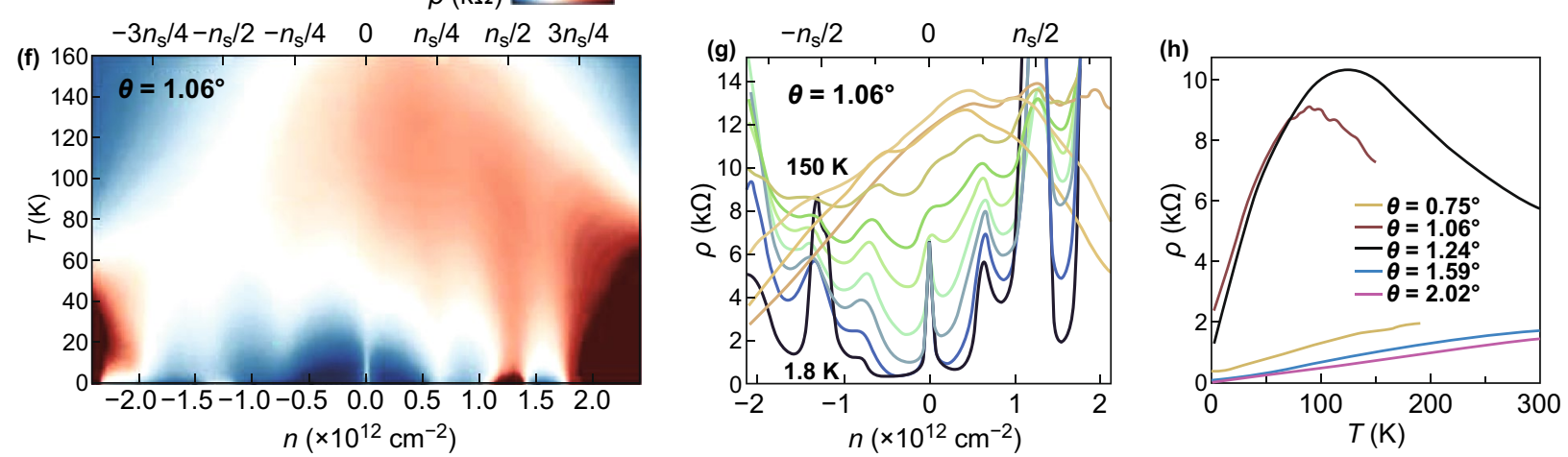

Fig. 8 a Variation in temperature-dependent conductivity, $\sigma$, of the tMLG device at $90 \mathrm{~K}$ to $273 \mathrm{~K}$ temperature range, and blue and red lines are power law and linear fit to the curves in the metallic region and semiconducting region, respectively. b Temporal variation in the photocurrent with the intermittent enlightenment of light source measured at $50 \mathrm{mV}$. c Photoresponse variation indicated by the varying of the current (left) and the resistance (right), measured at different temperatures [134]. d Normalized current distributions of four tBLG domains with different twisting angles. e Interlayer conductance of tBLG with different twisting angles. The orange curve and the red curve indicate a phonon-assisted transport mechanism with and without considering the renormalization of the Fermi velocity, respectively [135]. f Temperature-dependent resistivity $(\rho)$ of tBLG device. g Resistivity $(\rho)$ as a function of carrier density at selected temperatures in tBLG device, $\mathbf{h} \rho(T)$ recorded in tBLG devices with different twisting angles near $-n_{\mathrm{s}} / 2$ filling [137]. Adapted with permission from Refs. [134, 135, 137]

temperature regimes are noticeable by the different behavior of resistivity $(\rho(T))$ depending on the carrier density and twisting angle.

\section{Superconductivity}

The unusual superconducting behavior of different materials has been studied broadly for the last decades. The weak interlayer interaction creates the interlayer coupling in tBLG, and the strength of interlayer coupling as well as twisting angles equally affects the Fermi velocity and the VHSs of tBLG, which makes the novel electronic state of tBLG, which is different from those in SLG [138-140]. The twisting angle among the layers of bilayer graphene decides the degree of interlayer coupling and plays a decisive role in its electronic properties [140]. Recently, Cao et al. [141] reported the unconventional superconductivity in the magic-angle graphene superlattices. The representative 
device structure of the encapsulated tBLG is shown in Fig. 9a. The mini-Brillouin zone is built from the variation present among the two $K$ or $K^{\prime}$ wave vectors for the two graphene monolayers (Fig. 9b). The interlayer hybridization takes place between the Dirac cones in each valley, where the intervalley interactions are intensely suppressed $[120,142]$. The longitudinal resistance $\left(R_{\mathrm{xx}}\right)$, as a function of temperature $(T(\mathrm{~K}))$ for two tBLG devices with $1.16^{\circ}$ and $1.05^{\circ}$ twisting angles, demonstrated zero resistance at 70 $\mathrm{mK}$ revealing the superconducting state (Fig. 9c). The critical temperature $\left(T_{\mathrm{c}}\right)$ determined using a resistance of $50 \%$ of the non-superconducting state value was about 1.7 and $0.5 \mathrm{~K}$ for the two tBLG devices. The two-probe conductance versus carrier density at zero magnetic fields and a

(a)
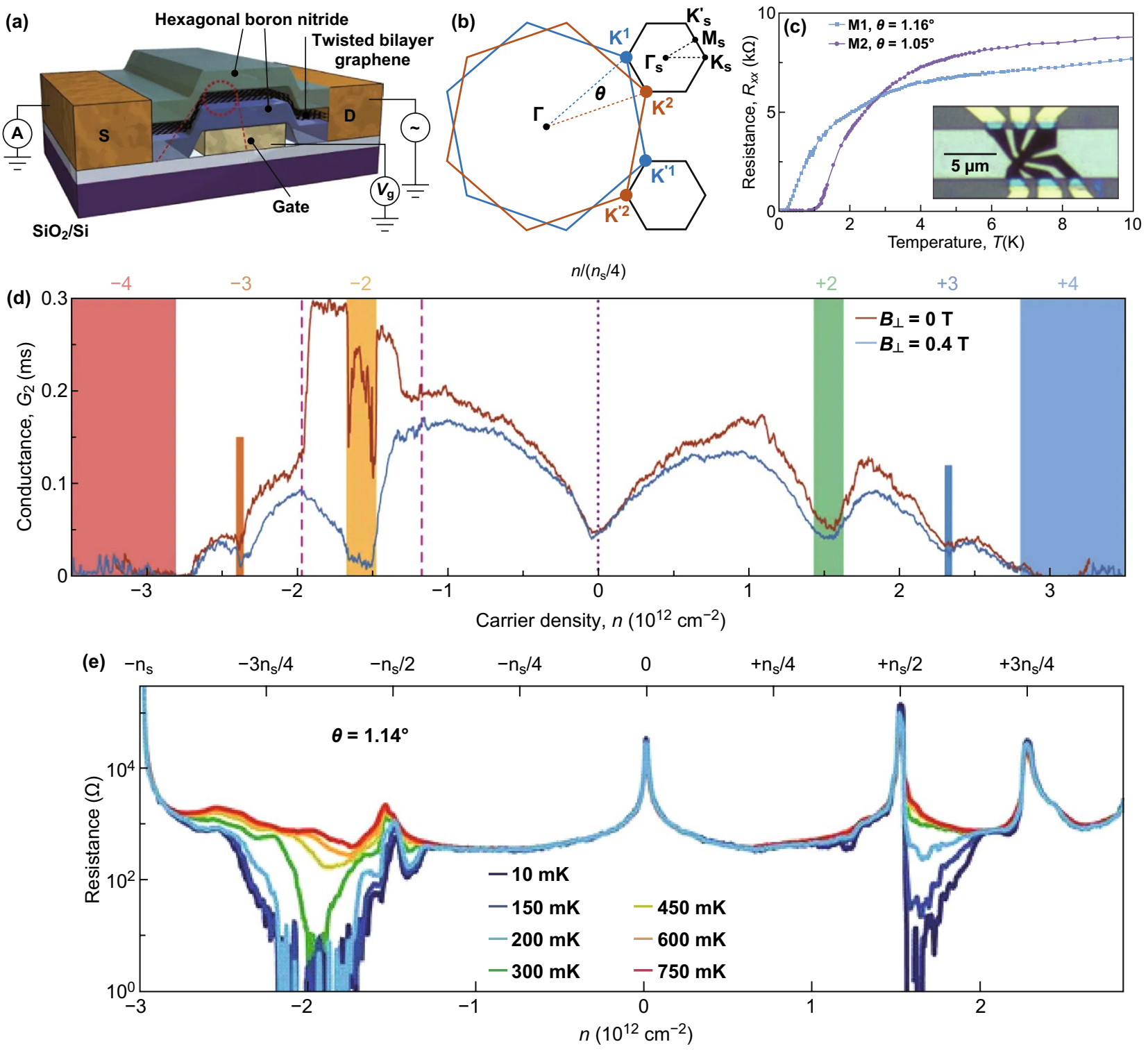

Fig. 9 a Schematic of the tBLG and hexagonal boron nitride-sandwiched device. b The mini-Brillouin zone is made from the difference between the two $K$ (or $K^{\prime}$ ) wave vectors for the two graphene layers [43]. c Four-probe resistance $R_{\mathrm{xx}}$ determined in two devices $M 1$ and $M 2$ with twisting angle of $1.16^{\circ}$ and $1.05^{\circ}$, respectively, and the optical image of the device is shown in inset where the darker region is the patterned tBLG. d Two-probe conductance $\left(G_{2}=I / V_{\text {bias }}\right)$ of the device M1 calculated in zero magnetic field (red) and at a vertical field of $B_{\perp}=0.4 \mathrm{~T}$ (blue) [141]. e Temperature dependence of the resistance over the density range required to fill the moire unit cell [143]. Adapted with permission from Refs. [43, 141, 143]. (Color figure online) 
$0.4 \mathrm{~T}$ perpendicular magnetic field of $M 1$ device is shown in Fig. 9d, which clearly shows the V-shaped conductance created from the renormalized Dirac cones of the tBLG band structure at charge neutrality point $(n=0)$. It also shows the states of insulating at the superlattice bandgaps $n= \pm n_{\mathrm{s}}$. The insulating state at $\pm 3.2 \times 10^{12} \mathrm{~cm}^{-2}$ is as a result of the presence of single-particle bandgaps in a band structure, as well as the observed conductance minima connected with many body gaps [43]. At $70 \mathrm{mK}$ temperature and $-1.3 \times 10^{12}$ to $-1.9 \times 10^{12} \mathrm{~cm}^{-2}$ electrons per unit cell, the conductance was significantly high for nil magnetic fields than in the vertical 0.4 T magnetic field $\left(B_{\perp}\right)$, which reveals the presence of superconductivity at the magic angle. The density-dependent resistance of the $\mathrm{tBLG}$ device with a $1.14^{\circ}$ twisting angle plotted almost over the complete flat band density range is shown in Fig. 9e [143]. The tBLG device showed lower charge carrier inhomogeneity $\left(\delta n<2 \times 10^{10} \mathrm{~cm}^{-2}\right)$, and at the magic angle $(\theta=\sim 1.1)$, the resultant hybridization and moiré superlattice among the graphene layers caused the development of a remote flat band at the charge neutrality point (CNP) [120, 143]. In the flat band, the resistive states are observed at the charge neutrality point (CNP) and $\pm n_{\mathrm{s}} / 2$ and $+3 n_{\mathrm{s}} / 4$. The superconductivity regions emerge in both electron- and hole-doped regions at $\sim 10 \mathrm{mK}$ base temperature with the dropping of resistance to zero. However, for $\pm n_{\mathrm{s}} / 2$ densities, below the base temperature, no sign of superconductivity is observed. The superconductivity appears considerably absent or weak on the lower-density side and extra stronger in the higher-density side of the insulator in both bands.

Recently, Codecido et al. [144] reported both insulating state and superconductivity in a tBLG structure at a $\sim 0.93^{\circ}$ twisting angle. The $\sim 0.93^{\circ}$ twisting angle is $15 \%$ small than the previously reported magic angle $\left(\theta=1.1 \pm 0.1^{\circ}\right)[43$, $139,140]$. The magnetic field $(B)$ and longitudinal resistance $\left(R_{\mathrm{xx}}\right)$ versus an elongated gate voltage $\left(V_{\mathrm{g}}\right)$ range at a $1.7 \mathrm{~K}$ temperature (Landau fan pattern) are shown in Fig. 10a. The satellite peak is observed at $V_{\mathrm{g}}= \pm 0.85 \mathrm{~V}$, as the lowerenergy moiré bands are filling at densities $n_{\mathrm{m}}= \pm 4$ (where $n_{\mathrm{m}}$ is the number of charges per moiré unit cell) $[43,141]$. The resistance peak appears at $V_{\mathrm{g}}=0.43 \mathrm{~V}$ from which an alongside set of Landau levels emanate. The peak on the half-filling and the twofold degeneracy of Landau levels illustrate the breaking of the symmetry of spin valley [145] and the development of a novel quasi-particle Fermi surface. At $n_{\mathrm{m}}=0, \pm 4$, and +2 , the resistance peaks are clearly
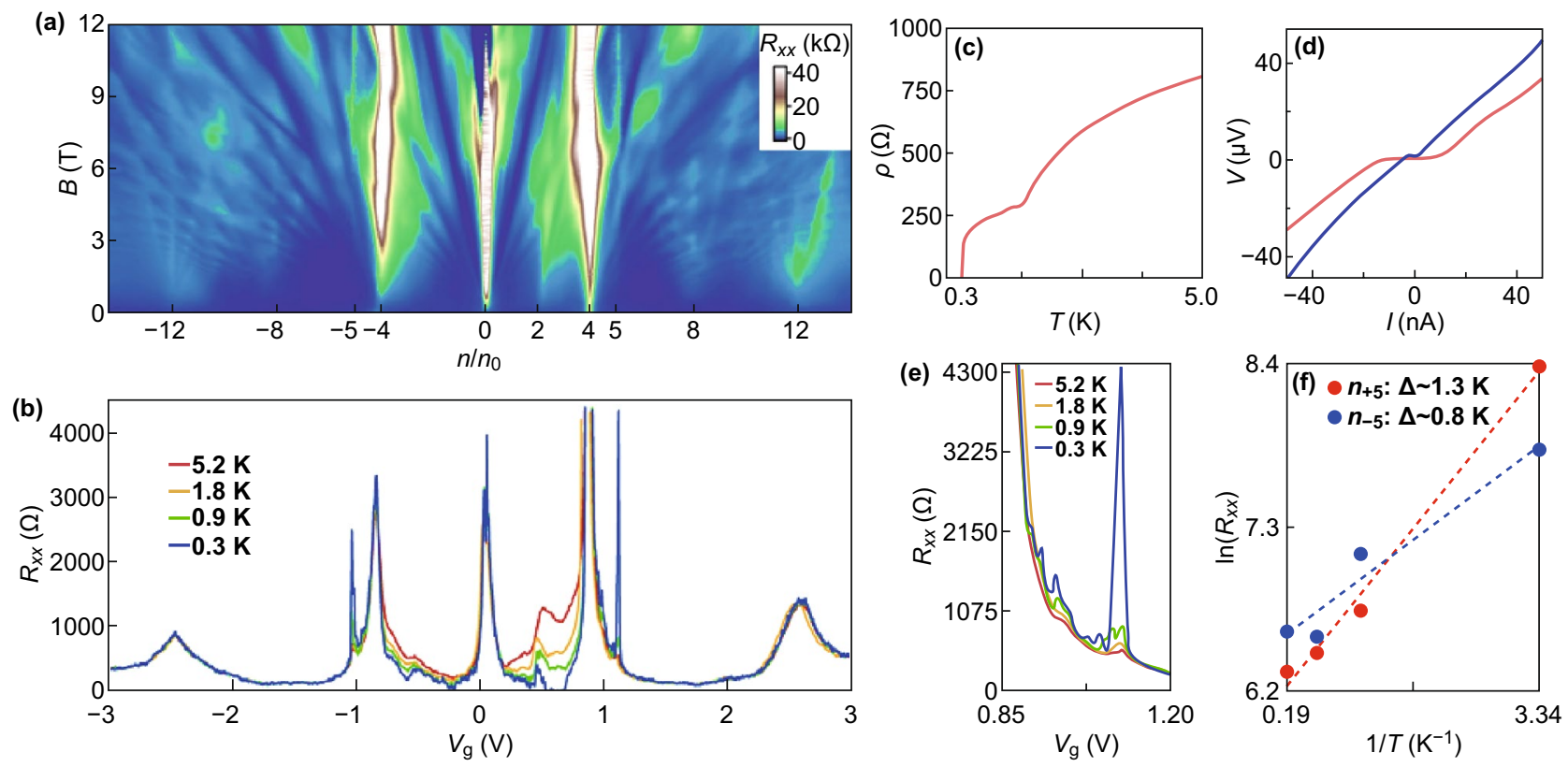

Fig. 10 a $R_{\mathrm{xx}}$ versus magnetic field $B$ and gate voltage $V_{\mathrm{g}}$, showing a Landau fan pattern. $\mathbf{b} R_{\mathrm{xx}}\left(V_{\mathrm{g}}\right)$ at different temperatures. $\mathbf{c} \rho$ versus temperature when the density is tuned to the superconducting phase. d Voltage-current characteristics at $T=280 \mathrm{mK}$ and $V_{\mathrm{g}}=0.58 \mathrm{~V}$ (red) and $0.50 \mathrm{~V}$ (blue). e Temperature dependence of the resistance peak. f Arrhenius plot of resistance [144]. Adapted with permission from Ref. [144]. (Color figure online) 
observed in $R_{\mathrm{xx}}\left(V_{\mathrm{g}}\right)$ at $B=0$ and for different temperatures (0.28 to $5.2 \mathrm{~K}$ ) (Fig. 10b). The $R_{\mathrm{xx}}$ is zero at $T=280 \mathrm{mK}$, for $0.51<V_{\mathrm{g}}<0.65$ revealing the development of superconductivity [43]. The development of superconductivity and conceivably penetrating superconducting regions might be responsible for the increase in $R_{\mathrm{xx}}$ with the increase in temperature at $n_{\mathrm{m}}=2$. As the temperature $(T)$ decreases, $\rho$ decreases to zero with the two consecutive steep successions at $T \sim 0.3 \mathrm{~K}$ and $T \sim 1.5 \mathrm{~K}$ (Fig. 10c); it could be associated with non-Planckian dissipation of the extraordinary metal states [146]. The voltage-current $(V-I)$ curves at two descriptive densities $\left(V_{\mathrm{g}}=0.58 \mathrm{~V}\right.$ and $\left.V_{\mathrm{g}}=0.50 \mathrm{~V}\right)$ are shown in Fig. 10d. The maximum value of the critical current $\left(I_{\mathrm{c}}\right)$ is observed at $V_{\mathrm{g}}=0.58 \mathrm{~V}$ and observed the supercurrent for an elongated range of density with $I_{\mathrm{c}}(\sim 1$ to $15 \mathrm{nA})$. The temperature dependence of the resistance peaks is nearly invisible, as the temperature is enhanced above $\sim 5 \mathrm{~K}$ (Fig. 10d). The Arrhenius plot of resistance with $\sim 1 \mathrm{~K}$ energy gap is shown in Fig. 10f. The peaks at $n_{\mathrm{m}} \approx \pm 5$ are unlikely to initiate from the angular disorder and single-particle gap as a result of an alignment between graphene and hBN [147, 148]; these features are uncertainly attributed to the development of a novel interrelated insulating state. $\theta=0.93^{\circ}$ is the lowest twisting angle reported till to the date for $\mathrm{BLG}$ devices showing superconductivity and insulating state.

\section{Conclusions}

The objective of this review paper is to provide detailed information regarding the fabrication of twisted bilayer graphene (tBLG) via different methods, its properties, as well as its technological applicability. The tBLG-related research field has developed at an enormous speed. The prominent tBLG fabrication methods such as micromechanical exfoliation, CVD, graphene flake pickup, CRS, stacking methods, and their unique properties are summarized in the initial sections. The control over the twisting of two graphene layers is the major challenge in the fabrication of tBLG. The twisted bilayer graphene (tBLG) is a novel arrangement, which shows the basic properties are different from those of the stacked bilayer graphene. The variation in line width and position of the $\left(\mathrm{ZO}^{\prime}\right)_{L}$ mode illustrates the influence of the twisting angle-dependent electronic band overlaps, onto the Raman spectrum. The continuous variation in optical and electrical properties of tBLG is strongly dependent on the twisting angle $(\theta)$ among the two graphene layers. We believe that the development and variation in the optical properties of tBLG would be extensively used in the future in the field of optoelectronics. The tBLG devices displayed non-monotonous conductivity variation, which reveals a semiconductor to metallic transition. The superconducting properties observed in $\mathrm{tBLG}$ are due to the electron interactions, which can distinctly influence the properties of moiré superlattices at higher densities and smaller twisting angles. There are quite a few challenges that are related to achieving a control over the twisting of two graphene layers for the development in the fabrication and characterization of twisted bilayer graphene (tBLG). It is expected that theoretical studies will be published in the future to search for novel superconducting and insulating phases of tBLG at a lower temperature.

Acknowledgements This work was supported by the Basic Science Research Program (2017K1A3A1A1907045513) through the National Research Foundation of Korea (NRF) and also by the DGIST R\&D programs (20-CoENT-01, 20-BT-06), funded by the Ministry of Science and ICT.

Open Access This article is licensed under a Creative Commons Attribution 4.0 International License, which permits use, sharing, adaptation, distribution and reproduction in any medium or format, as long as you give appropriate credit to the original author(s) and the source, provide a link to the Creative Commons licence, and indicate if changes were made. The images or other third party material in this article are included in the article's Creative Commons licence, unless indicated otherwise in a credit line to the material. If material is not included in the article's Creative Commons licence and your intended use is not permitted by statutory regulation or exceeds the permitted use, you will need to obtain permission directly from the copyright holder. To view a copy of this licence, visit http://creativecommons.org/licenses/by/4.0/.

\section{References}

1. K.S. Novoselov, A.K. Geim, S.V. Morozov, D. Jiang, Y. Zhang, S.V. Dubonos, I.V. Grigorieva, A.A. Firsov, Electric field effect in atomically thin carbon films. Science 306(5696), 666-669 (2004). https://doi.org/10.1126/scien ce. 1102896

2. M. Tahriri, M. Del Monico, A. Moghanian, M.T. Yaraki, R. Torres, A. Yadegari, L. Tayebi, Graphene and its derivatives: opportunities and challenges in dentistry. Mater. Sci. Eng. C 102, 171-185 (2019). https://doi.org/10.1016/j. msec.2019.04.051 
3. Z. Peng, Z. Yan, Z. Sun, J.M. Tour, Direct growth of bilayer graphene on $\mathrm{SiO}_{2}$ substrates by carbon diffusion through nickel. ACS Nano 5(10), 8241-8247 (2011). https://doi. org/10.1021/nn202923y

4. A.I. Cocemasov, D.L. Nika, A.A. Balandin, Phonons in twisted bilayer graphene. Phys. Rev. B 88, 035428 (2013). https://doi.org/10.1103/PhysRevB.88.035428

5. K.S. Novoselov, A.K. Geim, S.V. Morozov, D. Jiang, M.I. Katsnelson et al., Two-dimensional gas of massless Dirac fermions in graphene. Nature 438, 197-200 (2005). https:// doi.org/10.1038/nature04233

6. Y.B. Zhang, Y.W. Tan, H.L. Stormer, P. Kim, Experimental observation of the quantum Hall effect and Berry's phase in graphene. Nature 438, 201-204 (2005). https://doi. org/10.1038/nature04235

7. M. Freitag, H.Y. Chiu, M. Steiner, V. Perebeinos, P. Avouris, Thermal infrared emission from biased graphene. Nat. Nanotechnol. 5, 497-501 (2010). https://doi.org/10.1038/NNANO .2010 .90

8. C. Xu, H. Li, K. Banerjee, Modeling, analysis, and design of graphene nano-ribbon interconnects. IEEE Trans. Electron Dev. 56, 8 (2009). https://doi.org/10.1109/TED.2009.20242 54

9. W. Liu, S. Kraemer, D. Sarkar, H. Li, P.M. Ajayan, K. Banerjee, Controllable and rapid synthesis of high-quality and large-areabernal stacked bilayer graphene using chemical vapor deposition. Chem. Mater. 26(2), 907-915 (2014). https://doi.org/10.1021/cm4021854

10. S.Y. Dai, Y. Xiang, D.J. Srolovitz, Twisted bilayer graphene: moiré with a twist. Nano Lett. 16, 5923-5927 (2016). https ://doi.org/10.1021/acs.nanolett.6b02870

11. J. Hicks, M. Sprinkle, K. Shepperd, F. Wang, Symmetry breaking in commensurate graphene rotational stacking: comparison of theory and experiment. Phys. Rev. B 83, 205403 (2011). https://doi.org/10.1103/PhysRevB.83.205403

12. E.S. Morell, P. Vargas, Charge redistribution and interlayer coupling in twisted bilayer graphene under electric fields. Phys. Rev. B 84, 195421 (2011). https://doi.org/10.1103/ PhysRevB.84.195421

13. G. Tarnopolsky, A.J. Kruchkov, A. Vishwanath, Origin of magic angles in twisted bilayer graphene. Phys. Rev. Lett. 122, 106405 (2019). https://doi.org/10.1103/PhysRevLet t. 122.106405

14. M. Anđelković, L. Covaci, F.M. Peeters, DC conductivity of twisted bilayer graphene: angle-dependent transport properties and effects of disorder. Phys. Rev. Mater. 2, 034004 (2018). https://doi.org/10.1103/PhysRevMaterials.2.034004

15. E.J. Mele, Commensuration and interlayer coherence in twisted bilayer graphene. Phys. Rev. B 81, 161405(R) (2010). https://doi.org/10.1103/PhysRevB.81.161405

16. B.L. Huang, C.P. Chuu, M.F. Lin, Asymmetry-enriched electronic and optical properties of bilayer graphene. Sci. Rep. 9, 859 (2019). https://doi.org/10.1038/s41598-018-37058-9

17. C.Y. Lin, J.Y. Wu, Y.H. Chiu, C.P. Chang, M.F. Lin, Stacking-dependent magnetoelectronic properties in multilayer graphene. Phys. Rev. B 90, 205434 (2014). https://doi. org/10.1103/PhysRevB.90.205434

18. D.S. Lee, C. Riedl, T. Beringer, A.H.C. Neto, K.V. Klitzing, U. Starke, J.H. Smet, Quantum hall effect in twisted bilayer graphene. Phys. Rev. Lett. 107, 216602 (2011). https://doi. org/10.1103/PhysRevLett.107.216602

19. C.C. Lu, Y.C. Lin, Z. Liu, C.H. Yeh, K. Suenaga, P.W. Chiu, Twisting bilayer graphene superlattices. ACS Nano 7(3), 2587-2594 (2013). https://doi.org/10.1021/nn3059828

20. J.M.B. Lopes dos Santos, N.M.R. Peres, A.H.C. Neto, Graphene bilayer with a twist: electronic structure. Phys. Rev. Lett. 99, 256802 (2007). https://doi.org/10.1103/PhysRevLet t.99.256802

21. A.O. Sboychakov, A.L. Rakhmanov, A.V. Rozhkov, F. Nori, Electronic spectrum of twisted bilayer graphene. Phys. Rev. B 92, 075402 (2015). https://doi.org/10.1103/PhysR evB.92.075402

22. W. Yan, W.Y. He, Z.D. Chu, M.X. Liu, L. Meng et al., Strain and curvature induced evolution of electronic band structures in twisted graphene bilayer. Nat. Commun. 4, 2159 (2013). https://doi.org/10.1038/ncomms3159

23. J.S. Alden, A.W. Tsen, P.Y. Huang, R. Hovden, L. Brown, J.W. Park, D.A. Muller, P.L. McEuen, Strain solitons and topological defects in bilayer graphene. PNAS 110(28), 11256-11260 (2013). https://doi.org/10.1073/pnas.13093 94110

24. K. Kim, M. Yankowitz, B. Fallahazad, S. Kang, H.C.P. Movva et al., van der Waals heterostructures with high accuracy rotational alignment. Nano Lett. 16(3), 1989-1995 (2016). https://doi.org/10.1021/acs.nanolett.5b05263

25. L.J. Yin, H. Jiang, J.B. Qiao, L. He, Direct imaging of topological edge states at a bilayer graphene domain wall. Nat. Commun. 7, 11760 (2016). https://doi.org/10.1038/ncomm s11760

26. L. Huder, A. Artaud, T.L. Quang, G.T. de Laissardière, A.G.M. Jansen, G. Lapertot, C. Chapelier, V.T. Renard, Electronic spectrum of twisted graphene layers under heterostrain. Phys. Rev. Lett. 120, 156405 (2018). https://doi.org/10.1103/ PhysRevLett.120.156405

27. J.B. Qiao, L.J. Yin, L. He, Twisted graphene bilayer around the first magic angle engineered by heterostrain. Phys. Rev. B 98, 235402 (2018). https://doi.org/10.1103/PhysR evB.98.235402

28. L. Liao, H. Wang, H. Peng, J.B. Yin, A.L. Koh, Y.L. Chen, Q. Xie, H.L. Peng, Z.F. Liu, van Hove singularity enhanced photochemical reactivity of twisted bilayer graphene. Nano Lett. 15(8), 5585-5589 (2015). https://doi.org/10.1021/acs. nanolett.5b02240

29. C.R. Woods, L. Britnell, A. Eckmann, R.S. Ma, J.C. Lu, H.M. Guo et al., Commensurate-incommensurate transition in graphene on hexagonal boron nitride. Nat. Phys. 10, 451-456 (2014). https://doi.org/10.1038/NPHYS2954

30. Q. Ye, J. Wang, Z.B. Liu, Z.C. Deng, X.T. Kong et al., Polarization-dependent optical absorption of graphene under total internal reflection. Appl. Phys. Lett. 102, 021912 (2013). https://doi.org/10.1063/1.4776694 
31. R.W. Havener, Y.F. Liang, L. Brown, L. Yang, J. Park, Van Hove singularities and excitonic effects in the optical conductivity of twisted bilayer graphene. Nano Lett. 14, 3353-3357 (2014). https://doi.org/10.1021/nl500823k

32. M.D. Corato, C. Cocchi, D. Prezzi, M.J. Caldas, E. Molinari, A. Ruini, Optical properties of bilayer graphene nanoflakes. J. Phys. Chem. C 118(40), 23219-23225 (2014). https://doi. org/10.1021/jp504222m

33. S.K. Jain, V. Juričić, G.T. Barkema, Structure of twisted and buckled bilayer graphene. 2D Mater. 4, 015018 (2017). https ://doi.org/10.1088/2053-1583/4/1/015018

34. S. Shallcross, S. Sharma, O.A. Pankratov, Quantum interference at the twist boundary in graphene. Phys. Rev. Lett. 101, 056803 (2008). https://doi.org/10.1103/PhysRevLet t.101.056803

35. R. de Gail, M.O. Goerbig, F. Guinea, G. Montambaux, A.H.C. Neto, Topologically protected zero modes in twisted bilayer graphene. Phys. Rev. B 84, 045436 (2011). https://doi. org/10.1103/PhysRevB.84.045436

36. R. Bistritzer, A.H. MacDonald, Moiré butterflies in twisted bilayer graphene. Phys. Rev. B 84, 035440 (2011). https://doi. org/10.1103/PhysRevB.84.035440

37. G.T. de Laissardie`re, D. Mayou, L. Magaud, Localization of dirac electrons in rotated graphene bilayers. Nano Lett. 10, 804-808 (2010). https://doi.org/10.1021/n1902948m

38. L. Meng, W. Yan, L.J. Yin, Z.D. Chu, Y.F. Zhang, L. Feng, R. Dou, J. Nie, Tuning the interlayer coupling of the twisted bilayer graphene by molecular adsorption. J. Phys. Chem. C 118, 6462-6466 (2014). https://doi.org/10.1021/jp4109915

39. W. Yan, M.X. Liu, R.F. Dou, L. Meng, L. Feng et al., Angledependent van Hove Singularities in a slightly twisted graphene bilayer. Phys. Rev. Lett. 109, 126801 (2012). https:// doi.org/10.1103/PhysRevLett.109.126801

40. V.H. Nguyen, P. Dollfus, Strain-induced modulation of Dirac cones and van Hove singularities in twisted graphene bilayer. 2D Mater. 2, 035005 (2015). https://doi.org/10.1088/20531583/2/3/035005

41. J.D.S.Y. Taniguchi, A. Yacoby, P. Jarillo-Herrero, Quantum hall effect, screening, and layer-polarized insulating states in twisted bilayer graphene. Phys. Rev. Lett. 108, 076601 (2012). https://doi.org/10.1103/PhysRevLett.108.076601

42. M. Zarenia, I. Yudhistira, S. Adam, G. Vignale, Enhanced hydrodynamic transport in near magic angle twisted bilayer graphene. Phys. Rev. B 101, 045421 (2020). https://doi. org/10.1103/PhysRevB.101.045421

43. Y. Cao, V. Fatemi, A. Demir, S. Fang, S.L. Tomarken et al., Correlated insulator behaviour at half-filling in magic-angle graphene superlattices. Nature 556, 80-84 (2018). https://doi. org/10.1038/nature26154

44. B. Lian, Z.J. Wang, B.A. Bernevig, Twisted bilayer graphene: a phonon-driven superconductor. Phys. Rev. Lett. 122, 257002 (2019). https://doi.org/10.1103/PhysRevLet t.122.257002

45. N.F.Q. Yuan, L. Fu, Model for the metal-insulator transition in graphene superlattices and beyond. Phys. Rev. B 98,
045103 (2018). https://doi.org/10.1103/PhysRevB.98.04510 3

46. H.C. Po, L.J. Zou, A. Vishwanath, T. Senthil, Origin of Mott insulating behavior and superconductivity in twisted bilayer graphene. Phys. Rev. X 8, 031089 (2018). https://doi. org/10.1103/PhysRevX.8.031089

47. C. Xu, L. Balents, Topological superconductivity in twisted multilayer graphene. Phys. Rev. Lett. 121, 087001 (2018). https://doi.org/10.1103/PhysRevLett.121.087001

48. B. Roy, V. Juričić, Unconventional superconductivity in nearly flat bands in twisted bilayer graphene. Phys. Rev. B 99, 121407(R) (2019). https://doi.org/10.1103/PhysR evB.99.121407

49. G.E. Volovik, Graphite, graphene and the flat band superconductivity. JETP Lett. 107, 516 (2018). https://doi. org/10.1134/S0021364018080052

50. B. Padhi, C. Setty, P.W. Phillips, Doped twisted bilayer graphene near magic angles: proximity to Wigner crystallization, not Mott insulation. Nano Lett. 18, 6175 (2018). https://doi. org/10.1021/acs.nanolett.8b02033

51. J.F. Dodaro, S.A. Kivelson, Y. Schattner, X.Q. Sun, C. Wang, Phases of a phenomenological model of twisted bilayer graphene. Phys. Rev. B 98, 075154 (2018). https:// doi.org/10.1103/PhysRevB.98.075154

52. G. Baskaran, Theory of emergent Josephson lattice in neutral twisted bilayer graphene (moire is different). arXiv :1804.00627

53. F.C. Wu, A.H. MacDonald, I. Martin, Theory of phononmediated superconductivity in twisted bilayer graphene. Phys. Rev. Lett. 121, 257001 (2018). https://doi.org/10.1103/ PhysRevLett.121.257001

54. H. Isobe, N.F.Q. Yuan, L. Fu, Unconventional superconductivity and density waves in twisted bilayer graphene. Phys. Rev. X 8, 041041 (2018). https://doi.org/10.1103/PhysR evX.8.041041

55. T.Y. Huang, L.F. Zhang, T.X. Ma, Antiferromagnetically ordered mott insulator and $d+i d$ superconductivity in twisted bilayer graphene: a quantum monte carlo study. Sci. Bull. 64, 310 (2019). https://doi.org/10.1016/j.scib.2019.01.026

56. Y.Z. You, A. Vishwanath, Superconductivity from valley fluctuations and approximate $\mathrm{SO}(4)$ symmetry in a weak coupling theory of twisted bilayer graphene. NPJ Quantum Mater. 4, 16 (2019). https://doi.org/10.1038/s41535-019-0153-4

57. X.C. Wu, K.A. Pawlak, C.M. Jian, C.K. Xu, Emergent superconductivity in the weak Mott insulator phase of bilayer graphene Moiŕe superlattice. arXiv:1805.06906

58. Y.H. Zhang, D. Mao, Y. Cao, P.J. Herrero, T. Senthil, Nearly flat chern bands in moiré superlattices. Phys. Rev. B 99, 075127 (2019). https://doi.org/10.1103/PhysRevB.99.075127

59. J. Kang, O. Vafek, Maximally localized Wannier states, and a low-energy model for twisted bilayer graphene narrow bands. Phys. Rev. X 8, 031088 (2018). https://doi.org/10.1103/ PhysRevX.8.031088

60. M. Koshino, N.F.Q. Yuan, T. Koretsune, M. Ochi, K. Kuroki, L. Fu, Maximally localized Wannier orbitals and the extended 
Hubbard model for twisted bilayer graphene. Phys. Rev. X 8 , 031087 (2018). https://doi.org/10.1103/PhysRevX.8.031087

61. D.M. Kennes, J. Lischner, C. Karrasch, Strong correlations and $d+i d$ superconductivity in twisted bilayer graphene. Phys. Rev. B 98, 241407(R) (2018). https://doi.org/10.1103/ PhysRevB.98.241407

62. L. Zhang, Lowest-energy moire band formed by Dirac zero modes in twisted bilayer graphene. Sci. Bull. 64, 495 (2019). https://doi.org/10.1016/j.scib.2019.03.010

63. J.M. Pizarro, M.J. Calderón, E. Bascones, The nature of correlations in the insulating states of twisted bilayer graphene. J. Phys. Commun. 3, 035024 (2019). https://doi. org/10.1088/2399-6528/ab0fa9

64. F. Guineaa, N.R. Walet, Electrostatic effects, band distortions, and superconductivity in twisted graphene bilayers. Proc. Natl. Acad. Sci. U. S. A. 115, 13174 (2018). https:// doi.org/10.1073/pnas.1810947115

65. L.J. Zou, H.C. Po, A. Vishwanath, T. Senthil, Band structure of twisted bilayer graphene: emergent symmetries, commensurate approximants, and Wannier obstructions. Phys. Rev. B 98, 085435 (2018). https://doi.org/10.1103/PhysR evB.98.085435

66. M.P. Lima, J.E. Padilha, R.B. Pontes, A. Fazzio, A.J.R. Silva, Stacking-dependent transport properties in few-layers graphene. Solid State Commun. 250, 70-74 (2017). https://doi. org/10.1016/j.ssc.2016.11.012

67. C.X. Cong, T. Yu, Enhanced ultra-low-frequency interlayer shear modes in folded graphene layers. Nat. Commun. 5, 4709 (2014). https://doi.org/10.1038/ncomms5709

68. K.N. Kudin, B. Ozbas, H.C. Schniepp, R.K. Prud'homme, I.A. Aksay, R. Car, Raman spectra of graphite oxide and functionalized graphene sheets. Nano Lett. 8(1), 36-41 (2008). https://doi.org/10.1021/n1071822y

69. K.S. Novoselov, D. Jiang, F. Schedin, T.J. Booth, V.V. Khotkevich, S.V. Morozov, A.K. Geim, Two-dimensional atomic crystals. PNAS 102(30), 10451-10453 (2005). https://doi. org/10.1073/pnas.0502848102

70. S. Stankovich, D.A. Dikin, R.D. Piner, K.A. Kohlhaas, A. Kleinhammes et al., Synthesis of graphene-based nanosheets via chemical reduction of exfoliated graphite oxide. Carbon 45, 1558-1565 (2007). https://doi.org/10.1016/j.carbo n.2007.02.034

71. C. Virojanadara, M. Syväjarvi, R. Yakimova, L.I. Johansson, A.A. Zakharov, T. Balasubramanian, Homogeneous large-area graphene layer growth on $6 \mathrm{H}-\mathrm{SiC}(0001)$. Phys. Rev. B 78, 245403 (2008). https://doi.org/10.1103/PhysR evB.78.245403

72. C. Berger, Z.M. Song, X.B. Li, X.S. Wu, N. Brown et al., Electronic confinement and coherence in patterned epitaxial graphene. Science 312(5777), 1191-1196 (2006). https://doi. org/10.1126/science. 1125925

73. X. Li, W.W. Cai, J. An, S. Kim, J. Nah et al., Large-area synthesis of high-quality and uniform graphene films on copper foils. Science 324(5932), 1312-1314 (2009). https://doi. org/10.1126/science. 1171245
74. Y.C. Chen, W.H. Lin, W.S. Tseng, C.C. Chen, G.R. Rossman, C.D. Chen, Y.S. Wu, N.C. Yeh, Direct growth of mmsize twisted bilayer graphene by plasma-enhanced chemical vapor deposition. Carbon 156, 212-224 (2020). https://doi. org/10.1016/j.carbon.2019.09.052

75. J.T. Robinson, S.W. Schmucker, C.B. Diaconescu, J.P. Long, J.C. Culbertson, T. Ohta, A.L. Friedman, T.E. Beechem, Electronic hybridization of large-area stacked graphene films. ACS Nano 7(1), 637-644 (2013). https://doi.org/10.1021/ nn304834p

76. K.S. Kim, Y. Zhao, H. Jang, S.Y. Lee, J.M. Kim et al., Largescale pattern growth of graphene films for stretchable transparent electrodes. Nature 457, 706-710 (2009). https://doi. org/10.1038/nature07719

77. A. Reina, X.T. Jia, J. Ho, D. Nezich, H. Son et al., Large area, few-layer graphene films on arbitrary substrates by chemical vapor deposition. Nano Lett. 9(1), 30-35 (2009). https://doi. org/10.1021/n1801827v

78. S. Bae, H. Kim, Y. Lee, X.F. Xu, J.S. Park et al., Roll-to-roll production of 30-inch graphene films for transparent electrodes. Nat. Nanotechnol. 5, 574-578 (2010). https://doi. org/10.1038/NNANO.2010.132

79. K. Kim, A. DaSilva, S.Q. Huang, B. Fallahazad, S. Larentis et al., Tunable moiré bands and strong correlations in smalltwist-angle bilayer graphene. PNAS 114(13), 3364-3369 (2017). https://doi.org/10.1073/pnas.1620140114

80. L. Wang, I. Meric, P.Y. Huang, Q. Gao, Y. Gao et al., One dimensional electrical contact to a two-dimensional material. Science 342(6158), 614-617 (2013). https://doi.org/10.1126/ science. 1244358

81. X.D. Chen, W. Xin, W.S. Jiang, Z.B. Liu, Y.S. Chen, J.G. Tian, High-precision twist-controlled bilayer and trilayer graphene. Adv. Mater. 28, 2563-2570 (2016). https://doi. org/10.1002/adma.201505129

82. B. Wang, M. Huang, N.Y. Kim, B.V. Cunning, Y. Huang et al., Controlled folding of single crystal graphene. Nano Lett. 17, 1467-1473 (2017). https://doi.org/10.1021/acs.nanol ett.6b04459

83. L. Ju, Z.W. Shi, N. Nair, Y. Lv, C.H. Jin et al., Topological valley transport at bilayer graphene domain walls. Nature 520, 650-655 (2015). https://doi.org/10.1038/nature14364

84. A. Thomson, S. Chatterjee, S. Sachdev, M.S. Scheurer, Triangular antiferromagnetism on the honeycomb lattice of twisted bilayer graphene. Phys. Rev. B 98, 075109 (2018). https://doi. org/10.1103/PhysRevB.98.075109

85. T. Ohta, A. Bostwick, T. Seyller, K. Horn, E. Rotenberg, Controlling the electronic structure of bilayer graphene. Science 313(5789), 951-954 (2006). https://doi.org/10.1126/scien ce. 1130681

86. J.B. Yin, H. Wang, H. Peng, Z.J. Tan, L. Liao et al., Selectively enhanced photocurrent generation in twisted bilayer graphene with van Hove singularity. Nat. Commun. 7, 10699 (2016). https://doi.org/10.1038/ncomms10699

87. S. Shallcross, S. Sharma, O. Pankratov, Emergent momentum scale, localization, and van Hove singularities in the graphene 
twist bilayer. Phys. Rev. B 87, 245403 (2013). https://doi. org/10.1103/PhysRevB.87.245403

88. S. Shallcross, S. Sharma, E. Kandelaki, O.A. Pankratov, Electronic structure of turbostratic graphene. Phys. Rev. B 81, 1 (2010). https://doi.org/10.1103/PhysRevB.81.165105

89. W. Landgraf, S. Shallcross, K. Türschmann, D. Weckbecker, O. Pankratov, Electronic structure of twisted graphene flakes. Phys. Rev. B 87, 075433 (2013). https://doi.org/10.1103/ PhysRevB.87.075433

90. T. Ohta, J.T. Robinson, P.J. Feibelman, A. Bostwick, E. Rotenberg, T.E. Beechem, Evidence for interlayer coupling and moiré periodic potentials in twisted bilayer graphene. Phys. Rev. Lett. 109, 186807 (2012). https://doi. org/10.1103/PhysRevLett.109.186807

91. W. Yan, L. Meng, M.X. Liu, J.B. Qiao, Z.D. Chu et al., Angle-dependent van Hove singularities and their breakdown in twisted graphene bilayers. Phys. Rev. B 90, 115402 (2014). https://doi.org/10.1103/PhysRevB.90.115402

92. A. Luican, G.H. Li, A. Reina, J. Kong, R.R. Nair, K.S. Novoselov, A.K. Geim, E.Y. Andrei, Single-layer behavior and its breakdown in twisted graphene layers. Phys. Rev. Lett. 106, 126802 (2011). https://doi.org/10.1103/PhysR evLett.106.126802

93. S. Coh, L.Z. Tan, S.G. Louie, M.L. Cohen, Theory of the Raman spectrum of rotated double-layer graphene. Phys. Rev. B 88, 165431 (2013). https://doi.org/10.1103/PhysR evB.88.165431

94. K. Sato, R. Saito, C.X. Cong, T. Yu, M.S. Dresselhaus, Zone folding effect in Raman G-band intensity of twisted bilayer graphene. Phys. Rev. B 86, 125414 (2012). https:// doi.org/10.1103/PhysRevB.86.125414

95. P. Poncharal, A. Ayari, T. Michel, J.L. Sauvajol, Raman spectra of misoriented bilayer graphene. Phys. Rev. B 78, 113407 (2008). https://doi.org/10.1103/PhysR evB.78.113407

96. Z.H. Ni, Y.Y. Wang, T. Yu, Y.M. You, Z.X. Shen, Reduction of fermi velocity in folded graphene observed by resonance Raman spectroscopy. Phys. Rev. B 77, 235403 (2008). https ://doi.org/10.1103/PhysRevB.77.235403

97. Y.Y. Wang, Z.H. Ni, L. Liu, Y.H. Liu, C.X. Cong et al., Stacking-dependent optical conductivity of bilayer graphene. ACS Nano 4(7), 4074-4080 (2010). https://doi.org/10.1021/nn100 4974

98. I. Brihuega, P. Mallet, H. González-Herrero, G. Trambly de Laissardière, M.M. Ugeda et al., Unraveling the intrinsic and robust nature of van Hove singularities in twisted bilayer graphene by scanning tunneling microscopy and theoretical analysis. Phys. Rev. Lett. 109, 196802 (2012). https://doi. org/10.1103/PhysRevLett.109.196802

99. F. Symalla, S. Shallcross, I. Beljakov, K. Fink, W. Wenzel, V. Meded, Band-gap engineering with a twist: formation of intercalant superlattices in twisted graphene bilayers. Phys. Rev. B 91, 205412 (2015). https://doi.org/10.1103/PhysR evB.91.205412

100. D. Wong, Y. Wang, J. Jung, S. Pezzini, A.M. DaSilva et al., Crommie, local spectroscopy of moiré-induced electronic structure in gate-tunable twisted bilayer graphene. Phys. Rev. B 92, 155409 (2015). https://doi.org/10.1103/PhysR evB.92.155409

101. J.B. Wu, M.L. Lin, X. Cong, H.N. Liua, P.H. Tan, Raman spectroscopy of graphene-based materials and its applications in related devices. Chem. Soc. Rev. 47, 1822 (2018). https:// doi.org/10.1039/c6cs00915h

102. H. Nishi, Y. Matsushita, A. Oshiyama, Band-unfolding approach to moiré-induced band-gap opening and Fermi level velocity reduction in twisted bilayer graphene. Phys. Rev. B 95, 085420 (2017). https://doi.org/10.1103/PhysR evB.95.085420

103. K. Uchida, S. Furuya, J.I. Iwata, A. Oshiyama, Atomic corrugation and electron localization due to moiré patterns in twisted bilayer graphenes. Phys. Rev. B 90, 155451 (2014). https://doi.org/10.1103/PhysRevB.90.155451

104. A. Ferrari, D. Basko, Raman spectroscopy as a versatile tool for studying the properties of graphene. Nat. Nano 8, 235246 (2013). https://doi.org/10.1038/NNANO.2013.46

105. L.M. Malard, M.A. Pimenta, G. Dresselhaus, M.S. Dresselhaus, Raman spectroscopy in graphene. Phys. Rep. 473, 51-87 (2009). https://doi.org/10.1016/j.physrep.2009.02.003

106. L.M. Malard, J. Nilsson, D.C. Elias, J.C. Brant, F. Plentz, E.S. Alves, A.H. Castro Neto, M.A. Pimenta, Probing the electronic structure of bilayer graphene by Raman scattering. Phys. Rev. B 76, 201401(R) (2007). https://doi.org/10.1103/ PhysRevB.76.201401

107. A.C. Ferrari, J.C. Meyer, V. Scardaci, C. Casiraghi, M. Lazzeri et al., Raman spectrum of graphene and graphene layers. Phys. Rev. Lett. 97, 187401 (2006). https://doi. org/10.1103/PhysRevLett.97.187401

108. R. Saito, A. Jorio, A.G. Souza Filho, G. Dresselhaus, M.S. Dresselhaus, M.A. Pimenta, Probing phonon dispersion relations of graphite by double resonance Raman scattering. Phys. Rev. Lett. 88, 027401 (2001). https://doi.org/10.1103/ PhysRevLett.88.027401

109. C. Thomsen, S. Reich, Double resonant Raman scattering in graphite. Phys. Rev. Lett. 85(24), 5214-5217 (2000). https:// doi.org/10.1103/PhysRevLett.85.5214

110. V. Carozo, C.M. Almeida, E.H.M. Ferreira, L.G. Cancado, C.A. Achete, A. Jorio, Raman signature of graphene superlattices. Nano Lett. 11, 4527-4534 (2011). https://doi. org/10.1021/nl201370m

111. J.B. Wu, X. Zhang, M. Ijäs, W.P. Han, X.F. Qiao et al., Resonant Raman spectroscopy of twisted multilayer graphene. Nat. Commun. 5, 5309 (2014). https://doi.org/10.1038/ ncomms6309

112. J. Campos-Delgado, L.G. Cançado, C.A. Achete, A. Jorio, J.P. Raskin, Raman scattering study of the phonon dispersion in twisted bilayer graphene. Nano Res. 6, 269-274 (2013). https://doi.org/10.1007/s12274-013-0304-Z

113. J.B. Wu, H. Wang, X.L. Li, H.L. Peng, P.H. Tan, Raman spectroscopic characterization of stacking configuration and interlayer coupling of twisted multilayer graphene grown by chemical vapor deposition. Carbon 110, 225-231 (2016). https://doi.org/10.1016/j.carbon.2016.09.006 
114. C.H. Lui, L.M. Malard, S. Kim, G. Lantz, F.E. Laverge, R. Saito, T.F. Heinz, Observation of layer-breathing mode vibrations in few-layer graphene through combination Raman scattering. Nano Lett. 12, 5539-5544 (2012). https://doi. org/10.1021/nl302450s

115. R. He, T.F. Chung, C. Delaney, C. Keiser, L.A. Jauregui et al., Observation of low energy Raman modes in twisted bilayer graphene. Nano Lett. 13, 3594-3601 (2013). https:// doi.org/10.1021/n14013387

116. A.K. Gupta, Y.J. Tang, V.H. Crespi, P.C. Eklund, Nondispersive Raman $D$ band activated by well-ordered interlayer interactions in rotationally stacked bilayer graphene. Phys. Rev. B 82, 241406(R) (2010). https://doi.org/10.1103/ PhysRevB.82.241406

117. A. Jorio, M. Kasperczyk, N. Clark, E. Neu, P. Maletinsky, A. Vijayaraghavan, L. Novotny, Optical-phonon resonances with saddle-point excitons in twisted-bilayer graphene. Nano Lett. 14, 5687-5692 (2014). https://doi.org/10.1021/ $\mathrm{nl} 502412 \mathrm{~g}$

118. R.W. Havener, H. Zhuang, L. Brown, R.G. Hennig, J. Park, Angle-resolved Raman imaging of interlayer rotations and interactions in twisted bilayer graphene. Nano Lett. 12, 3162-3167 (2012). https://doi.org/10.1021/nl301137k

119. J. Hass, F. Varchon, J.E. Millán-Otoya, M. Sprinkle, N. Sharma et al., Why multilayer graphene on $4 \mathrm{H}-\mathrm{SiC}(0001)$ behaves like a single sheet of graphene. Phys. Rev. Lett. 100, 125504 (2008). https://doi.org/10.1103/PhysRevLet t.100.125504

120. R. Bistritzer, A.H. MacDonald, Moiré bands in twisted double-layer graphene. PNAS 108(30), 12233-12237 (2011). https://doi.org/10.1073/pnas.1108174108

121. J.M.B. Lopes dos Santos, N.M.R. Peres, A.H. Castro Neto, Continuum model of the twisted graphene bilayer. Phys. Rev. B 86, 155449 (2012). https://doi.org/10.1103/PhysR evB.86.155449

122. M. Koshino, Stacking-dependent optical absorption in multilayer graphene. N. J. Phys. 15, 015010 (2013). https://doi. org/10.1088/1367-2630/15/1/015010

123. Z.Q. Li, E.A. Henriksen, Z. Jiang, Z. Hao, M.C. Martin, P. Kim, H.L. Stormer, D.N. Basov, Band structure asymmetry of bilayer graphene revealed by infrared spectroscopy. Phys. Rev. Lett. 102, 037403 (2009). https://doi. org/10.1103/PhysRevLett.102.037403

124. K.F. Mak, M.Y. Sfeir, J.A. Misewich, T.F. Heinz, The evolution of electronic structure in few-layer graphene revealed by optical spectroscopy. PNAS 107(34), 14999-15004 (2010). https://doi.org/10.1073/pnas.1004595107

125. Y.B. Zhang, T.T. Tang, C. Girit, Z. Hao, M.C. Martin et al., Direct observation of a widely tunable bandgap in bilayer graphene. Nature 459, 820-823 (2009). https://doi. org/10.1038/nature08105

126. H.A. Le, V.N. Do, Electronic structure and optical properties of twisted bilayer graphene calculated via time evolution of states in real space. Phys. Rev. B 97, 125136 (2018). https://doi.org/10.1103/PhysRevB.97.125136
127. K. Yu, N.V. Luan, T. Kim, J. Jeon, J. Kim, P. Moon, Y.H. Lee, E.J. Choi, Gate tunable optical absorption and band structure of twisted bilayer graphene. Phys. Rev. B 99, 241405(R) (2019). https://doi.org/10.1103/PhysRevB.99.241405

128. C.J. Tabert, E.J. Nicol, Optical conductivity of twisted bilayer graphene. Phys. Rev. B 87, 121402(R) (2013). https ://doi.org/10.1103/PhysRevB.87.121402

129. P. Moon, M. Koshino, Optical absorption in twisted bilayer graphene. Phys. Rev. B 87, 205404 (2013). https://doi. org/10.1103/PhysRevB.87.205404

130. H. Patel, L. Huang, C.J. Kim, J. Park, M.W. Graham, Stacking angle-tunable photoluminescence from interlayer exciton states in twisted bilayer graphene. Nat. Commun. 10, 1445 (2019). https://doi.org/10.1038/s41467-019-09097-X

131. F. Xia, T. Mueller, R.G. Mojarad, M. Freitag, Y.M. Lin, J. Tsang, V. Perebeinos, P. Avouris, Photocurrent imaging and efficient photon detection in a graphene transistor. Nano Lett. 9(3), 1039-1044 (2009). https://doi.org/10.1021/n1803 3812

132. T. Mueller, F. Xia, M. Freitag, J. Tsang, P. Avouris, Role of contacts in graphene transistors: a scanning photocurrent study. Phys. Rev. B 79, 245430 (2009). https://doi. org/10.1103/PhysRevB.79.245430

133. U. Mogera, R. Dhanya, R. Pujar, C. Narayana, G.U. Kulkarni, Highly decoupled graphene multilayers: turbostraticity at its best. J. Phys. Chem. Lett. 6, 4437-4443 (2015). https://doi. org/10.1021/acs.jpclett.5b02145

134. U. Mogera, S. Walia, B. Bannur, M. Gedda, G.U. Kulkarni, Intrinsic nature of graphene revealed in temperature-dependent transport of twisted multilayer graphene. J. Phys. Chem. C 121, 13938-13943 (2017). https://doi.org/10.1021/acs. jpcc.7b04068

135. Z.W. Yu, A. Song, L.Z. Sun, Y. Li, L. Gao et al., Understanding interlayer contact conductance in twisted bilayer graphene. Small (2019). https://doi.org/10.1002/smll.20190 2844

136. L. Brown, R. Hovden, P. Huang, M. Wojcik, D.A. Muller, J. Park, Twinning and twisting of tri- and bilayer graphene. Nano Lett. 12, 1609-1615 (2012). https://doi.org/10.1021/ nl204547y

137. H. Polshyn, M. Yankowitz, S.W. Chen, Y.X. Zhang, K. Watanabe, T. Taniguchi, C.R. Dean, A.F. Young, Large linearin-temperature resistivity in twisted bilayer graphene. Nat. Phys. 15, 1011-1016 (2019). https://doi.org/10.1038/s4156 7-019-0596-3

138. L.J. Yin, J.B. Qiao, W.X. Wang, W.J. Zuo, W. Yan et al., Landau quantization and Fermi velocity renormalization in twisted graphene bilayers. Phys. Rev. B 92, 201408(R) (2015). https://doi.org/10.1103/PhysRevB.92.201408

139. J. Wang, X. Mu, L. Wang, M. Sun, Properties and applications of new superlattice: twisted bilayer graphene. Mater. Today Phys. 9, 100099 (2019). https://doi.org/10.1016/j. mtphys.2019.100099

140. B. Deng, B.B. Wang, N. Li, R. Li, Y. Wang et al., Interlayer decoupling in $30^{\circ}$ twisted bilayer graphene quasicrystal. ACS 
Nano 14, 1656-1664 (2020). https://doi.org/10.1021/acsna no. $9 \mathrm{~b} 07091$

141. Y. Cao, V. Fatemi, S. Fang, K. Watanabe, T. Taniguchi, E. Kaxiras, P.J. Herrero, Unconventional superconductivity in magic-angle graphene superlattices. Nature 556, 43-50 (2018). https://doi.org/10.1038/nature26160

142. Y. Cao, J.Y. Luo, V. Fatemi, S. Fang, J.D. Sanchez-Yamagishi et al., Superlattice-induced insulating states and valley-protected orbits in twisted bilayer graphene. Phys. Rev. Lett. 117, 116804 (2016). https://doi.org/10.1126/science.aav1910

143. M. Yankowitz, S.W. Chen, H. Polshyn, Y.X. Zhang, K. Watanabe et al., Tuning superconductivity in twisted bilayer graphene. Science 363(6431), 1059-1064 (2019). https://doi. org/10.1073/pnas.1108174108

144. E. Codecido, Q.Y. Wang, R. Koester, S. Che, H.D. Tian, Correlated insulating and superconducting states in twisted bilayer graphene below the magic. Sci. Adv. 5(9), eaaw9770 (2019). https://doi.org/10.1126/sciadv.aaw9770
145. F. Hu, S.R. Das, Y. Luan, T.F. Chung, Y.P. Chen, Z. Fei, Realspace imaging of the tailored plasmons in twisted bilayer graphene. Phys. Rev. Lett. 119, 247402 (2017). https://doi. org/10.1103/PhysRevLett.119.247402

146. Y. Cao, D. Chowdhury, D.R. Legrain, O.R. Bigorda, K. Watanabe et al., Strange metal in magic-angle graphene with near Planckian dissipation. Phys. Rev. Lett. 124, 076801 (2020). https://doi.org/10.1103/PhysRevLett.124.076801

147. L.A. Ponomarenko, R.V. Gorbachev, G.L. Yu, D.C. Elias, R. Jalil et al., Cloning of Dirac fermions in graphene superlattices. Nature 497, 594-597 (2013). https://doi.org/10.1038/ nature 12187

148. C.R. Dean, L. Wang, P. Maher, C. Forsythe, F. Ghahari et al., Hofstadter's butterfly and the fractal quantum Hall effect in moiré superlattices. Nature 497, 598-602 (2013). https://doi. org/10.1038/nature12186 